\title{
MARTINGALE REPRESENTATION PROCESSES AND APPLICATIONS IN THE MARKET VIABILITY UNDER INFORMATION FLOW EXPANSION
}

\author{
SHIQI SONG ${ }^{1}$
}

\begin{abstract}
When the martingale representation property holds, we call any local martingale which realizes the representation a representation process. There are two properties of the representation process which can greatly facilitate the computations under the martingale representation property. On the one hand, the representation process is not unique and there always exists a representation process which is locally bounded and has pathwise orthogonal components outside of a predictable thin set. On the other hand, the jump measure of a representation process satisfies the finite predictable constraint, which implies the martingale projection property. In this paper, we give a detailed account of these properties. As application, we will prove that, under the martingale representation property, the full viability of an expansion of market information flow implies the drift multiplier assumption.
\end{abstract}

\section{INTRODUCTION}

The present paper, jointly with $[20,22]$, takes part in a research program about the full viability problem (cf. Definition 5.2).

\subsection{The problem and the context}

Precisely, we consider a filtration $\mathbb{F}$ of sub- $\sigma$-algebras on a probability space and we consider all strictly positive $\mathbb{F}$ semimartingale $S$, which satisfies the no-arbitrage condition of the first kind $\left(\mathrm{NA}_{1}\right.$ in abbreviation, cf. [17] for definition) in $\mathbb{F}$. The pair $(\mathbb{F}, S)$ constitutes a financial market model, where $\mathbb{F}$ represents the information flow and $S$ represents the asset process. As the no-arbitrage condition $\mathrm{NA}_{1}$ ensures the utility optimization problems have solutions (cf. $[6,17])$, the models such as $(\mathbb{F}, S)$ are called viable market models. The market viability of $(\mathbb{F}, S)$ is a filtration-depending property. We are concerned in this paper with the filtration expansions which preserve fully the market viability, i.e., those filtrations $\mathbb{G}$ such that $\mathbb{G} \supset \mathbb{F}$ and all the asset process $S$ which is viable in $\mathbb{F}$ continue to be viable in $\mathbb{G}$ (the full viability).

The information change has always been a fundamental issue in market modeling. Since the work [9] on the insider trading problem, the study takes a direction of quantifying the information changes. For various reasons, agents who trade a same asset process may enjoy different streams of information. Different information leads to different trading strategy. For questions such as the insider trading or the risk hedging, it is essential to be able to compute the financial gains of the agents in terms of the variations of the information. This gain computation is not straightforward because of, notably, the difficulty to quantify the variation of an information change. On the other hand, by the no-arbitrage consideration, the gain computations are limited to the only case where the information changes preserve the market viability. In recent times, the class of the information changes which preserve the market viability has become a subject in itself. See, for example, the papers $[1,2,8,20]$. According

\footnotetext{
1 Laboratoire de Mathématiques et Modélisation d'Evry, Université d'Evry Val D'Essonne, France. shiqi.song@univ-evry.fr
}

(C) EDP Sciences, SMAI 2017 
to these papers, a major feature of that class is that the information changes satisfy some specific structural conditions. These structural conditions provide valuable means to quantify the information variation and to realize the corresponding gain computations.

A first study of the viability after an information change is [8]. It is followed by $[1,2,20]$. The present paper takes part in the same program of [20]. Following the classic settings of the initial or progressive enlargement of filtration, the papers $[1,2,8]$ provide conditions to have the full viability. The paper [20] succeeds to do the work beyond the classic settings, with a new necessary and sufficient condition for the full viability under the martingale representation property. The present paper establishes the structural implications of the full viability on the underlying filtration expansion $\mathbb{F} \subset \mathbb{G}$.

The proof of [20] involves peculiarly three properties, i.e., the finite predictable constraint property, the martingale projection property and the reconstruction of the representation process. The present paper is a detailed study of the three properties, with an application on the full viability. It is organized as follows.

\subsection{The contents}

The martingale projection property is defined for any (multi-dimensional) local martingale $M$ by the fact that, for any real local martingale $Y$ such that the (vector of) predictable dual projection $[Y, M]^{\mathbb{F} \cdot p}$ exists, there exists a predictable process $H$, integrable with respect to $M$, such that the predictable dual projection $[H \cdot M, M]^{\mathbb{F} \cdot p}$ exists and the identity

$$
[Y, M]^{\mathbb{F} \cdot p}=\left[{ }^{\top} H \cdot M, M\right]^{\mathbb{F} \cdot p}
$$

holds. The martingale projection property is very useful, for example, in the proof of [20, Theorem 4.3] or in the proof of Theorem 5.6 below. This property always holds, when $M$ is continuous. However, according to [3], the property does not always hold in the case of a purely discontinuous local martingale. The question is how to recognize a purely discontinuous local martingale $M$ which satisfies the martingale projection property.

It seems not very widely known, but the answer exists since Jacod [11], which consists to verify if the jump measure of $M$ satisfies the condition of finite predictable constraint (cf. Definition 3.1). (This last notion is therefore extracted from [11, Théorème 4.80] and a name is given to it because of its importance in the study of the full viability.) For the applications in this paper and in [20], we will extend the finite predictable constraint condition to any integer valued random measure $\mu$ and we will give in Section 3 a detailed analysis of the space

$$
\left\{W_{*}(\mu-\nu): W \text { is }(\mu-\nu) \text {-integrable in the sense of }[11]\right\}
$$

under that condition. The main result is Theorem 3.3. Specifications in the cases of accessible and respectively inaccessible time supports (cf. Section 3.1 for definition) are given in Section 3.3 and Section 3.4.

Usually the martingale representation property is mentioned to characterize a specific process (a Brownian motion, for example). But, in this paper, what is relevant is a stochastic basis having a martingale representation property, whatever the representation processes are. Yet more, we should make use of various different representation processes to make easier the computations under the martingale representation property. In Section 4 , based on the fact that the martingale representation property in the filtration $\mathbb{F}$ implies the finite conditional multiplicity condition of the filtration $\mathbb{F}$ (cf. [11] and [5] and Section 4.1 Lemma 4.4), we prove in Theorem 4.8 and Corollary 4.14 and Theorem 4.2 that, when the martingale representation property holds, it is always possible to reconstruct the representation process so that it becomes locally bounded and has pathwise orthogonal components outside of a predictable thin set.

One of the structural conditions obeyed by the filtration changes of full viability is the drift multiplier assumption (cf. Assumption 5.5). In application of the results in Section 3 and Section 4, we will prove in Theorem 5.6 that, under the martingale representation property in $\mathbb{F}$, the full viability of an information expansion $\mathbb{F} \subset \mathbb{G}$ implies the Hypothesis $\left(H^{\prime}\right.$ ) (cf. Section 5 for definition), and the corresponding drift operator satisfies the drift multiplier assumption. This means that every $\mathbb{F}$ local martingale $X$ is a $\mathbb{G}$ special semimartingale and there exist a common (multi-dimensional) $\mathbb{F}$ local martingale $N$ and a $\mathbb{G}$ predictable process $\varphi$ such that the drift 
part $\Gamma(X)$ of $X$ in $\mathbb{G}$ writes in the form

$$
(\nabla) \quad \Gamma(X)={ }^{\top} \varphi \cdot[N, X]^{\mathbb{F} \cdot p} \text {. }
$$

The main points of this result are (a) the $\mathbb{F}$ local martingale $N$ and the $\mathbb{G}$ predictable process $\varphi$ are common for all $\mathbb{F}$ local martingale $X$ and $(\mathbf{b})$ the predictable dual projection $[N, X]^{\mathbb{F} \cdot p}$ exists for all $\mathbb{F}$ local martingale $X$. The common existence of the pair $(\varphi, N)$ makes it a good means to quantify the variation of the information expansion $\mathbb{F} \subset \mathbb{G}$ (cf. [20] for a detailed formula). The drift multiplier assumption is a new notion in the literature of the theory of filtration enlargement. It has encountered serious questions about its relevance and its usefulness. Theorem 5.6 and the work [22] help to dispel the questions.

Section 5.2 presents some general consequences of the full viability assumption on an expansion of information flow $\mathbb{F} \subset \mathbb{G}$. For example, we will see that the $\mathbb{F}$ inaccessible stopping times remain inaccessible in $\mathbb{G}$, or that $A^{\mathbb{G} \cdot p}$ is absolutely continuous with respect to $A^{\mathbb{F} \cdot p}$ for any $\mathbb{F}$ adapted locally bounded increasing process $A$. These results are interesting in themselves. We also emphasize the benefits of working with the drift multiplier assumption in an enlarged filtration.

\subsection{N.B.}

Notice that in this introduction we have written the key notions in italic. This rule will be left out in the rest of the paper. Notice also that the assumption of the martingale representation property in $\mathbb{F}$ needs not mean that the market model $(\mathbb{F}, S)$ is complete.

\section{Notation AND CONVENTION}

We work on a probability space $(\Omega, \mathcal{A}, \mathbb{P})$ endowed with a filtration $\mathbb{F}=\left(\mathcal{F}_{t}\right)_{t \geq 0}$ of sub- $\sigma$-algebras of $\mathcal{A}$, satisfying the usual conditions. We employ the vocabulary of stochastic calculus as defined in $[10,11]$ with the specifications below.

Relations between random variables are to be understood almost sure relations. For a random variable $X$ and a $\sigma$-algebra $\mathcal{F}$, the expression $X \in \mathcal{F}$ means that $X$ is $\mathcal{F}$-measurable. The notation $\mathbf{L}^{p}(\mathbb{P}, \mathcal{F})$ denotes the space of $p$-times $\mathbb{P}$-integrable $\mathcal{F}$-measurable random variables.

By definition, $\Delta_{0} X=0$ for any càdlàg process $X$. A process $A$ with finite variation considered in this paper is automatically assumed càdlàg. We denote by $\mathrm{d} A$ the (signed) random measure that $A$ generates.

Different vector spaces $\mathbb{R}^{d}$ are used in the paper. An element $v$ in $\mathbb{R}^{d}$ is considered as a vertical vector. We denote its transposition by ${ }^{\top} v$. We denote (indifferently) the null vector by $\mathbf{0}$.

We deal with finite family of real processes $X=\left(X_{i}\right)_{1 \leq i \leq d}\left(d \in \mathbb{N}^{*}\right)$. It will be considered as process taking values in the vector space $\mathbb{R}^{d}$. To mention such an $X$, we say that $X$ is a $d$-dimensional process. In general we denote by $X_{i}$ the $i$ th component of the vector $X$. When $X$ is a semimartingale, we denote by $\left[X,{ }^{\top} X\right]$ the $d \times d$-dimensional matrix valued process whose components are $\left[X_{i}, X_{j}\right]$ for $1 \leq i, j \leq k$.

With respect to the filtration $\mathbb{F}$, the notation ${ }^{\mathbb{F} \cdot p} \bullet$ denotes the predictable projection, and the notation $\bullet$ F $p$ denotes the predictable dual projection.

For any $\mathbb{F}$ special semimartingale $X$, we can decompose $X$ in the form (see [10, Theorem 7.25]) :

$$
X=X_{0}+X^{m}+X^{v}, X^{m}=X^{c}+X^{d a}+X^{d i}
$$

where $X^{m}$ is the martingale part of $X$ and $X^{v}$ is the predictable part of finite variation of $X, X^{c}$ is the continuous martingale part, $X^{d a}$ is the part of compensated sum of accessible jumps, $X^{d i}$ is the part of compensated sum of totally inaccessible jumps. We recall that this decomposition of $X$ depends on the reference probability and the reference filtration. In the computations below we apply this notation system only for the decompositions in $\mathbb{F}$. We recall that every part of the decomposition of $X$, except $X_{0}$, is assumed null at $t=0$. 
In this paper we employ the notion of stochastic integral only with respect to the predictable processes. The stochastic integral are defined as 0 at $t=0$. We use a point ". to indicate the integrator process in a stochastic integral. For example, the stochastic integral of a real predictable process $H$ with respect to a real semimartingale $Y$ is denoted by $H \cdot Y$, while the expression ${ }^{\top} K\left(\cdot\left[X,{ }^{\top} X\right]\right) K$ denotes the process

$$
\int_{0}^{t} \sum_{i=1}^{k} \sum_{j=1}^{k}\left(K_{s}\right)_{i, s}\left(K_{s}\right)_{j, s} \mathrm{~d}\left[X_{i}, X_{j}\right]_{s}, t \geq 0,
$$

where $K$ is a $k$-dimensional predictable process and $X$ is a $k$-dimensional semimartingale. The expression ${ }^{\top} K\left(\cdot\left[X,{ }^{\top} X\right]\right) K$ respects the matrix product rule. The value at $t \geq 0$ of a stochastic integral will be denoted, for example, by ${ }^{\top} K\left(\cdot\left[X,{ }^{\top} X\right]\right) K_{t}$.

The notion of the stochastic integral with respect to a $d$-dimensional local martingale $X$ follows [11]. We say that a $d$-dimensional $\mathbb{F}$ predictable process is integrable with respect to $X$ under the probability $\mathbb{P}$ in the filtration $\mathbb{F}$, if the non decreasing process $\sqrt{{ }^{\top} H\left(\cdot\left[X,{ }^{\top} X\right]\right) H}$ is $(\mathbb{P}, \mathbb{F})$ locally integrable. For such an integrable process $H$, the stochastic integral ${ }^{\top} H \cdot X$ is well-defined and the bracket process of ${ }^{\top} H \cdot X$ can be computed using [11, Remarque(4.36) and Proposition(4.68)]. Note that two different predictable processes may produce the same stochastic integral with respect to $X$. In this case, we say that they are in the same equivalence class.

Again another notion of stochastic integral is needed, i.e., the stochastic integral with respect to a compensated integer valued random measure $\mu-\nu$. We refer to $[10,11,13]$ for its definition and the fundamental properties. In particular, we denote by $\mathcal{G}(\mathbb{F}, \mu)$ the space of $(\mu-\nu) *$-integrable $\mathbb{F}$ predictable functions. To distinguish the different type of stochastic integrals, we mention the stochastic integral with respect to a compensated integer valued random measure $\mu-\nu$ as stochastic ${ }_{*}$-integral, whilst the stochastic integral with respect to a semimartingale will be mentioned as stochastic --integral.

Warning. Notations may be reused in different parts of the paper for different meaning.

\section{Finite predictable constraint}

This section is devoted to the condition of finite predictable constraint for integer valued random measures. Recall that $\mathbb{F}$ is a filtration on the probability space $(\Omega, \mathcal{A}, \mathbb{P})$, satisfying the usual conditions.

\subsection{The definition}

We recall the basic vocabulary about integer valued random measures. Let $E$ be an Euclidean space. An $\mathbb{F}$ optional random measure $\mu$ on $\mathbb{R}_{+} \times E$ is said to be integer valued (cf. $[10,11]$ ), if there exists an $\mathbb{F}$ optional thin set $\mathrm{D}$ (the time support set) and an E-valued $\mathbb{F}$ optional process $\beta$ (the space location process) such that

$$
\mu[\mathrm{A}]=\sum_{s>0} \mathbb{1}_{\left\{\left(s, \beta_{s}\right) \in \mathrm{A}\right\}} \mathbb{1}_{\{s \in \mathrm{D}\}}, \forall \mathrm{A} \in \mathcal{B}\left(\mathbb{R}_{+} \times \mathrm{E}\right) .
$$

We make use of the results in [10, Chapiter XI section 1], also in [13, Chapiter II section 1]. In this paper, the integer valued random measures $\mu$ are always supposed to be $\sigma$-finite on the predictable $\sigma$-algebra and to have an $\mathbb{F}$ compensator $\nu$ satisfying

$$
\nu[\{0\} \times \mathrm{E}]=\nu\left[\mathbb{R}_{+} \times\{\mathbf{0}\}\right]=0,\left(|x|^{2} \wedge 1\right)_{*} \nu_{t}<\infty, t \in \mathbb{R}_{+} .
$$

Note that these conditions are satisfied by the jump measure of any semimartingale (cf. [13, Chapter II, Proposition 2.9]). Recall that $\mathcal{G}(\mathbb{F}, \mu)$ denotes the $(\mu-\nu)_{*}$-integrable predictable functions.

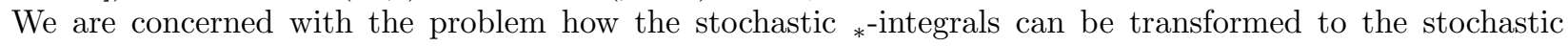
--integrals. Here is the notion which makes the transformation possible (cf. Theorem 3.3). 
Definition 3.1. We say that an integer valued $\mathbb{F}$ optional random measure $\mu$ satisfies the finite predictable constraint condition, if the space location process $\beta$ is confined in a finite $\mathbb{F}$ predictable constraint, i.e., if there exist a finite number (say $\mathrm{n}$ ) of $\mathrm{E}$-valued $\mathbb{F}$ predictable processes $\alpha_{k}, 1 \leq k \leq \mathrm{n}$, such that, at any time, the value of $\beta$ coincides with one of the values $\alpha_{k}$ or $\mathbf{0}$ :

$$
\beta \in\left\{\mathbf{0}, \alpha_{1}, \ldots, \alpha_{\mathrm{n}}\right\}
$$

Remark 3.2. Note that, in the case of a finite predictable constraint for $\beta$, we can modify the $E$-valued $\mathbb{F}$ predictable constraint processes $\alpha_{k}, 1 \leq k \leq \mathrm{n}$, to write

$$
\beta=\sum_{k=1}^{\mathrm{n}} \alpha_{k} \mathbb{1}_{\left\{\beta=\alpha_{k}\right\}}+\mathbf{0} \mathbb{1}_{\left\{\beta \neq \alpha_{k}, \forall k\right\}}
$$

We accept some set $\left\{\beta=\alpha_{k}\right\}$ empty.

\subsection{The main result}

We consider an integer valued random measure $\mu$ with its time support $\mathrm{D}$ and its space location process $\beta$ and its $\mathbb{F}$ compensator $\nu$. Suppose the finite predictable constraint condition with the constraint processes $\alpha_{k}, 1 \leq k \leq \mathrm{n}$. Let $e_{k}(1 \leq k \leq \mathrm{n})$ be a bounded continuous real function such that $e_{k}\left(\alpha_{k}\right) \neq 0$ on the time support set $\mathrm{D}$ of $\mu$, and $\left|e_{k}(x)\right| \leq c(|x| \wedge 1), x \in \mathrm{E}$, for some constant $c$.

Theorem 3.3. Suppose that $\mu$ satisfies the finite $\mathbb{F}$ predictable constraint condition with constraint processes $\alpha_{k}, 1 \leq k \leq \mathrm{n}$, satisfying identity (2). Suppose $\mathrm{D} \subset\{\beta \neq \mathbf{0}\}$. For $1 \leq k \leq \mathrm{n}$, let $e_{k}$ be the above defined functions.

(1) Let $u_{k}(s, x)=e_{k}(x) \mathbb{1}_{\left\{x=\alpha_{k, s}\right\}}, s \geq 0, x \in \mathrm{E}$, and $X_{k}=u_{k *}(\mu-\nu), 1 \leq k \leq \mathrm{n}$. Then, $X_{k}$ are well-defined locally bounded $\mathbb{F}$ local martingales.

(2) For an element $g \in \mathcal{G}(\mathbb{F}, \mu)$, let $\frac{g(\cdot, \alpha)}{\mathrm{e}(\alpha)} \mathbb{1}_{\{e(\alpha) \neq 0\}}$ denote the vector valued process composed of $g\left(\cdot, \alpha_{k}\right) \frac{1}{e_{k}\left(\alpha_{k}\right)} \mathbb{1}_{\left\{e_{k}\left(\alpha_{k}\right) \neq 0\right\}}, 1 \leq k \leq \mathrm{n}$. Then, $\frac{g(\cdot, \alpha)}{\mathrm{e}(\alpha)} \mathbb{1}_{\{e(\alpha) \neq 0\}}$ is integrable with respect to the vector valued process $X=\left(X_{k}\right)_{1 \leq k \leq \mathrm{n}}$.

(3) For any element $g \in \mathcal{G}(\mathbb{F}, \mu)$,

$$
g_{*}(\mu-\nu)={ }^{\top}\left(\frac{g(\cdot, \alpha)}{\mathrm{e}(\alpha)} \mathbb{1}_{\{e(\alpha) \neq 0\}}\right) \cdot X .
$$

We have the identity

$$
\left\{g_{*}(\mu-\nu): g \in \mathcal{G}(\mathbb{F}, \mu)\right\}=\left\{{ }^{\top} H \cdot X: H \text { is } X \text {-integrable }\right\} .
$$

Proof. Using the notations in [10, Definition 11.16], we must prove that the process $\left(\sqrt{\sum_{s \leq t} \widetilde{u}_{k, s}^{2}}, t \geq 0\right)$ is locally integrable, where

$$
\widetilde{u}_{k}=u_{k}(\cdot, \beta) \mathbb{1}_{\mathrm{D}}-\widehat{u}_{k} .
$$

We consider separately $\sum_{s \leq t, s \in \mathrm{D}} u_{k}\left(s, \beta_{s}\right)^{2}$ and $\sum_{s \leq t} \widehat{u}_{k, s}^{2}$. For any stopping time $T$ such that $\mathbb{E}\left[\left(|x|^{2} \wedge 1\right)_{*} \nu_{T}\right]<$ $\infty$, we have

$$
\mathbb{E}\left[\sum_{s \leq T, s \in \mathrm{D}} u_{k}\left(s, \beta_{s}\right)^{2}\right]=\mathbb{E}\left[u_{k *}^{2} \mu_{T}\right] \leq c^{2} \mathbb{E}\left[\left(|x|^{2} \wedge 1\right)_{*} \mu_{T}\right]=c^{2} \mathbb{E}\left[\left(x^{2} \wedge 1\right)_{*} \nu_{T}\right]<\infty
$$


On the other hand, we know that $\left\{\widehat{u}_{k} \neq 0\right\}$ is a predictable thin set J (cf. [10, Theorem 11.14]). Hence,

$$
\mathbb{E}\left[\sum_{s \leq T} \widehat{u}_{k, s}^{2}\right]=\mathbb{E}\left[\sum_{s \leq T, s \in \mathrm{J}} \widehat{u}_{k, s}^{2}\right] \leq \mathbb{E}\left[\sum_{s \leq T, s \in \mathrm{J}} \int_{[s] \times \mathbb{E}} u_{k}^{2} \mathrm{~d} \nu\right]=\mathbb{E}\left[\int_{[0, T] \times \mathbb{E}} \mathbb{1}_{J} u_{k}^{2} \mathrm{~d} \nu\right] \leq c^{2} \mathbb{E}\left[\int_{[0, T] \times \mathbb{E}}\left(|x|^{2} \wedge 1\right) \mathrm{d} \nu\right]<\infty .
$$

Because of the conditions in (1), we conclude that the process $\left(\sqrt{\sum_{s \leq t} \widetilde{u}_{k, s}^{2}}, t \geq 0\right)$ is locally integrable and the local martingale $X_{k}$ is well defined.

With identity (2), necessarily $\alpha_{k^{\prime}} \neq \alpha_{k}$ on $\left\{\beta \neq \mathbf{0}, \beta=\alpha_{k}\right\}$, for all $k^{\prime} \neq k$. As D $\subset\{\beta \neq \mathbf{0}\}$, the sets $\left\{s \in \mathrm{D}, \beta_{s}=\alpha_{k}\right\}, 1 \leq k \leq \mathrm{n}$, are mutually disjoint, and $\left\{s \in \mathrm{D}, \forall k, \beta_{s} \neq \alpha_{k}\right\}=\emptyset$. So, for a $g \in \mathcal{G}(\mathbb{F}, \mu)$, for any $\mathbb{F}$ stopping time $T$,

$$
g\left(T, \beta_{T}\right) \mathbb{1}_{\{T \in \mathrm{D}\}}=\sum_{k=1}^{\mathrm{n}} g\left(T, \alpha_{k, T}\right) \mathbb{1}_{\left\{\beta_{T}=\alpha_{k, T}\right\}} \mathbb{1}_{\{T \in \mathrm{D}\}} .
$$

We compute the jump at an $\mathbb{F}$ totally inaccessible stopping time $T$ on $\{T<\infty\}$.

$$
\begin{aligned}
& \Delta_{T}\left(g_{*}(\mu-\nu)\right)=g\left(T, \beta_{T}\right) \mathbb{1}_{\{T \in \mathrm{D}\}}=\sum_{k=1}^{\mathrm{n}} g\left(T, \alpha_{k, T}\right) \mathbb{1}_{\left\{\beta_{T}=\alpha_{k, T}\right\}} \mathbb{1}_{\{T \in \mathrm{D}\}} \\
= & \sum_{k=1}^{\mathrm{n}} g\left(T, \alpha_{k, T}\right) \frac{1}{e_{k}\left(\alpha_{k, T}\right)} \mathbb{1}_{\left\{e_{k}\left(\alpha_{k, T}\right) \neq 0\right\}} \Delta_{T} X_{k} .
\end{aligned}
$$

We compute next the jump at an $\mathbb{F}$ predictable stopping time $T$ on $\{T<\infty\}$.

$$
\begin{aligned}
& \Delta_{T}\left(g_{*}(\mu-\nu)\right) \\
= & g\left(T, \beta_{T}\right) \mathbb{1}_{\{T \in \mathrm{D}\}}-\mathbb{E}\left[g\left(T, \beta_{T}\right) \mathbb{1}_{\{T \in \mathrm{D}\}} \mid \mathcal{F}_{T-}\right] \\
= & \sum_{k=1}^{\mathrm{n}} g\left(T, \alpha_{k, T}\right) \mathbb{1}_{\left\{\beta_{T}=\alpha_{k, T}\right\}} \mathbb{1}_{\{T \in \mathrm{D}\}}-\mathbb{E}\left[\sum_{k=1}^{\mathrm{n}} g\left(T, \alpha_{k, T}\right) \mathbb{1}_{\left\{\beta_{T}=\alpha_{k, T}\right\}} \mathbb{1}_{\{T \in \mathrm{D}\}} \mid \mathcal{F}_{T-}\right] \\
= & \sum_{k=1}^{\mathrm{n}} g\left(T, \alpha_{k, T}\right)\left(\mathbb{1}_{\left\{\beta_{T}=\alpha_{k, T}\right\}} \mathbb{1}_{\{T \in \mathrm{D}\}}-\mathbb{E}\left[\mathbb{1}_{\left\{\beta_{T}=\alpha_{k, T}\right\}} \mathbb{1}_{\{T \in \mathrm{D}\}} \mid \mathcal{F}_{T-}\right]\right) \\
= & \sum_{k=1}^{\mathrm{n}=1} g\left(T, \alpha_{k, T}\right) \frac{1}{e_{k}\left(\alpha_{k, T}\right)}\left(e_{k}\left(\alpha_{k, T}\right) \mathbb{1}_{\left\{\beta_{T}=\alpha_{k, T}\right\}} \mathbb{1}_{\{T \in \mathrm{D}\}}-\mathbb{E}\left[e_{k}\left(\alpha_{k, T}\right) \mathbb{1}_{\left\{\beta_{T}=\alpha_{k, T}\right\}} \mathbb{1}_{\{T \in \mathrm{D}\}} \mid \mathcal{F}_{T-}\right]\right) \\
= & \sum_{k=1}^{\mathrm{n}} g\left(T, \alpha_{k, T}\right) \frac{1}{e_{k}\left(\alpha_{k, T}\right)}\left(e_{k}\left(\beta_{T}\right) \mathbb{1}_{\left\{\beta_{T}=\alpha_{k, T}\right\}} \mathbb{1}_{\{T \in \mathrm{D}\}}-\mathbb{E}\left[e_{k}\left(\beta_{T}\right) \mathbb{1}_{\left\{\beta_{T}=\alpha_{k, T}\right\}} \mathbb{1}_{\{T \in \mathrm{D}\}} \mid \mathcal{F}_{T-}\right]\right) \\
= & \sum_{k=1}^{\mathrm{n}} g\left(T, \alpha_{k, T}\right) \frac{1}{e_{k}\left(\alpha_{k, T}\right)} \mathbb{1}_{\left\{e_{k}\left(\alpha_{k, T}\right) \neq 0\right\}} \Delta_{T} X_{k} .
\end{aligned}
$$

We obtain the identity

$$
\Delta\left(g_{*}(\mu-\nu)\right)=\sum_{k=1}^{\mathrm{n}} g\left(\cdot, \alpha_{k}\right) \frac{1}{e_{k}\left(\alpha_{k}\right)} \mathbb{1}_{\left\{e_{k}\left(\alpha_{k}\right) \neq 0\right\}} \Delta X_{k} .
$$

This identity shows firstly that the process $\frac{g(\cdot, \alpha)}{\mathrm{e}(\alpha)} \mathbb{1}_{\{e(\alpha) \neq 0\}}$ is $X$-integrable. Secondly, by [10, Theorem 7.23], we have the equality

$$
g_{*}(\mu-\nu)={ }^{\top}\left(\frac{g(\cdot, \alpha)}{\mathrm{e}(\alpha)} \mathbb{1}_{\{e(\alpha) \neq 0\}}\right) \cdot X .
$$

Remark 3.4. Theorem 3.3 proves a possibility to represent the stochastic ${ }_{*}$-integrals in terms of the stochastic --integrals with respect to the local martingale $X$. We notice that the way Theorem 3.3 has been proved suggests the possibility to represent the stochastic ${ }_{*}$-integrals with different local martingales other than $X$. This is a very useful option, especially when the random measure $\mu$ is the jump measure of a local martingale $M$, and then one needs to write the representations with $M$ itself.

\subsection{Case of random measure with accessible time support}

In this section and the following one, we study the stochastic ${ }_{*}$-integrals, when the random measure $\mu$ is the jump measure of a local martingale $M$.

Consider an integer valued random measure $\mu$ with compensator $\nu$. We now cut the process $\beta$ into pieces in time and in space. Suppose that there exist a sequence of mutually avoiding $\mathbb{F}$ predictable stopping times 
$\left(T_{n}\right)_{1 \leq n<\mathrm{N}}(\mathrm{N} \leq \infty)$ such that the time support set is given by $\mathrm{D}=\left(\cup_{1 \leq n<\mathrm{N}}\left[T_{n}\right]\right) \cap\{\beta \neq \mathbf{0}\}$. We consider the family of random variables $\left(\beta_{T_{n}}, 1 \leq n<\mathrm{N}\right)$. Suppose in addition that there exist a positive integer $\mathrm{n}$ such that, for every $1 \leq n<\mathrm{N}$, there exist E-valued $\mathcal{F}_{T_{n}-}$ measurable $\alpha_{n, k}, 1 \leq k \leq \mathrm{n}$, and an $\mathcal{F}_{T_{n}}$ measurable partition $\left(A_{n, 1}, \ldots, A_{n, \mathrm{n}}\right)$ (possibly some empty sets) such that $\beta_{T_{n}}$ is cut into

$$
\beta_{T_{n}}=\sum_{k=1}^{\mathrm{n}} \alpha_{n, k} \mathbb{1}_{A_{n, k}} .
$$

Let $\left(a_{n}\right)_{1 \leq n<\mathrm{N}}$ be any series of non vanishing random variables such that $a_{n} \in \mathcal{F}_{T_{n}-}$ and the following expression

$$
Y_{k}=\sum_{n=1}^{\mathrm{N}-} a_{n}\left(\mathbb{1}_{A_{n, k}} \mathbb{1}_{\left[T_{n}, \infty\right)}-\left(\mathbb{1}_{A_{n, k}} \mathbb{1}_{\left[T_{n}, \infty\right)}\right)^{\mathbb{F} \cdot p}\right), 1 \leq k \leq \mathrm{n},
$$

defines an $\mathrm{n}$-dimensional $\mathbb{F}$ local martingale $Y$ (where $\mathrm{N}$ may be a finite integer and the upper bound $\mathrm{N}-$ means that the summation involves only the $n<\mathrm{N})$. Set $\alpha_{k}=\sum_{n=1}^{\mathrm{N}-} \alpha_{n, k} \mathbb{1}_{\left[T_{n}\right]}, 1 \leq k \leq \mathrm{n}$, and $G=\sum_{n=1}^{\mathrm{N}-} \frac{1}{a_{n}} \mathbb{1}_{\left[T_{n}\right]}$. For any $g \in \mathcal{G}(\mathbb{F}, \mu)$, denote by $g(\cdot, \alpha) \mathbb{1}_{\{\alpha \neq 0\}}$ the vector valued process $\left(g\left(\cdot, \alpha_{k}\right) \mathbb{1}_{\left\{\alpha_{k} \neq \mathbf{0}\right\}}\right)_{1 \leq k \leq \mathrm{n}}$.

Theorem 3.5. Under the above conditions, for any $g \in \mathcal{G}(\mathbb{F}, \mu), G g(\cdot, \alpha) \mathbb{1}_{\{\alpha \neq 0\}}$ is $Y$-integrable and

$$
g_{*}(\mu-\nu)=G^{\top} g(\cdot, \alpha) \mathbb{1}_{\{\alpha \neq 0\}} \cdot Y .
$$

Remark 3.6. If $\beta$ satisfies condition (3), (a version of) $\beta$ satisfies the finite predictable constraint.

Proof. For $g \in \mathcal{G}(\mathbb{F}, \mu)$, as in the proof of Theorem 3.3, we compute the jumps at one of the $\mathbb{F}$ predictable stopping times $T=T_{n}<\infty(1 \leq n<\mathrm{N})$.

$$
\begin{aligned}
& \Delta_{T}\left(g_{*}(\mu-\nu)\right) \\
= & g\left(T, \beta_{T}\right) \mathbb{1}_{\{T \in \mathrm{D}\}}-\mathbb{E}\left[g\left(T, \beta_{T}\right) \mathbb{1}_{\{T \in \mathrm{D}\}} \mid \mathcal{F}_{T-}\right] \\
= & g\left(T, \beta_{T}\right) \mathbb{1}_{\left\{\beta_{T} \neq \mathbf{0}\right\}}-\mathbb{E}\left[g\left(T, \beta_{T}\right) \mathbb{1}_{\left\{\beta_{T} \neq \mathbf{0}\right\}} \mid \mathcal{F}_{T-}\right] \\
= & \sum_{k=1}^{\mathrm{n}} g\left(T, \alpha_{k, T}\right) \mathbb{1}_{A_{n, k}} \mathbb{1}_{\left\{\alpha_{k, T} \neq \mathbf{0}\right\}}-\mathbb{E}\left[\sum_{k=1}^{\mathrm{n}} g\left(T, \alpha_{k, T}\right) \mathbb{1}_{A_{n, k}} \mathbb{1}_{\left\{\alpha_{k, T} \neq \mathbf{0}\right\}} \mid \mathcal{F}_{T-}\right] \\
= & \sum_{k=1}^{\mathrm{n}=1} g\left(T, \alpha_{k, T}\right) \mathbb{1}_{\left\{\alpha_{k, T} \neq \mathbf{0}\right\}}\left(\mathbb{1}_{A_{n, k}}-\mathbb{E}\left[\mathbb{1}_{A_{n, k}} \mid \mathcal{F}_{T-}\right]\right) \\
= & \sum_{k=1}^{\mathrm{n}=1} g\left(T, \alpha_{k, T}\right) \mathbb{1}_{\left\{\alpha_{k, T} \neq \mathbf{0}\right\}} \frac{1}{a_{n}} \Delta_{T} Y_{k} \\
= & \sum_{k=1}^{\mathrm{n}=1} g\left(T, \alpha_{k, T}\right) \mathbb{1}_{\left\{\alpha_{k, T} \neq \mathbf{0}\right\}} G_{T} \Delta_{T} Y_{k} .
\end{aligned}
$$

From this jump identity, we conclude that $G g(\cdot, \alpha) \mathbb{1}_{\{\alpha \neq 0\}}$ is $Y$-integrable, and, by [10, Theorem 7.23],

$$
g_{*}(\mu-\nu)=G^{\top}\left(g(\cdot, \alpha) \mathbb{1}_{\{\alpha \neq 0\}}\right) \cdot Y \text {. }
$$

Suppose, on top of the conditions in Theorem 3.5, that, for a $d$-dimensional $\mathbb{F}$ purely discontinuous local martingale $M, \mathrm{D}=\{\Delta M \neq \mathbf{0}\}$ and $\beta=\Delta M$. For a fixed $1 \leq n<\mathrm{N}$, let us view the $\mathcal{F}_{T_{n}-\text { measurable random }}$ variables as constants and consider the space $\mathfrak{L}_{n}$ of real functions generated by the indicators $\mathbb{1}_{A_{n, k}}, 1 \leq k \leq \mathrm{n}$. The space $\mathfrak{L}_{n}$ is of finite dimension and there exists a natural linear map from $\mathbb{R}^{\mathrm{n}}\left(\mathcal{F}_{T_{n}-}\right.$ measurable random variables being constant) onto the space $\mathfrak{L}_{n}$. Condition (3) says that the components $\Delta_{T_{n}} M_{i}, 1 \leq i \leq d$, of $\Delta_{T_{n}} M$ are elements in the space $\mathfrak{L}_{n}$ :

$$
\Delta_{T_{n}} M_{i}=\sum_{k=1}^{\mathrm{n}} \alpha_{n, i, k} \mathbb{1}_{A_{n, k}}
$$

where $\alpha_{n, i, k}$ denotes the $i$ th component of $\alpha_{n, k} \in \mathbb{R}^{d}$. Introduce the (vertical) vectors $\gamma_{n, i}$ of the components $\left(\alpha_{n, i, k}\right)_{1 \leq k \leq \mathrm{n}}\left(\gamma_{n, i}\right.$ being a representative of $\Delta_{T_{n}} M_{i}$ in $\left.\mathbb{R}^{\mathrm{n}}\right)$. Denote $p_{n, k}=\mathbb{E}\left[\mathbb{1}_{A_{n, k}} \mid \mathcal{F}_{T_{n}-}\right]$ and $p_{n}$ the (vertical) 
vectors of the components $\left(p_{n, k}\right)_{1 \leq k \leq n}$. We have for $1 \leq i \leq d$

$$
0=\mathbb{E}\left[\Delta_{T_{n}} M_{i} \mid \mathcal{F}_{T_{n}-}\right]=\sum_{k=1}^{\mathrm{n}} \alpha_{n, i, k} \mathbb{E}\left[\mathbb{1}_{A_{n, k}} \mid \mathcal{F}_{T_{n}-}\right]=\sum_{k=1}^{\mathrm{n}} \alpha_{n, i, k} p_{n, k}={ }^{\top} \gamma_{n, i} p_{n},
$$

i.e., $\gamma_{n, i}$ is orthogonal to $p_{n}$. Let $P_{n}=\sum_{k=1}^{\mathrm{n}} p_{n, k} \mathbb{1}_{A_{n, k}}$.

Theorem 3.7. Let $M$ be a d-dimensional $\mathbb{F}$ purely discontinuous local martingale such that $\mathrm{D}=\{\Delta M \neq \mathbf{0}\}$ and $\beta=\Delta M$ satisfy the conditions in Theorem 3.5. Suppose that the vectors $\gamma_{n, i}, 1 \leq i \leq d$, together with $p_{n}$ span the whole space $\mathbb{R}^{\mathrm{n}}$ (so that $\Delta_{T_{n}} M_{i}, 1 \leq i \leq d$, and $P_{n}$ generates $\mathfrak{L}_{n}$ and therefore $\mathrm{n} \leq d+1$ ). Then, for any $g \in \mathcal{G}(\mathbb{F}, \mu)$, there exists a matrix valued $\mathbb{F}$ predictable process $K$ such that $K g(\cdot, \alpha) \mathbb{1}_{\{\alpha \neq 0\}}$ is $M$-integrable and

We have the identity

$$
g_{*}(\mu-\nu)={ }^{\top}\left(K g(\cdot, \alpha) \mathbb{1}_{\{\alpha \neq 0\}}\right) \cdot M .
$$

$$
\left\{g_{*}(\mu-\nu): g \in \mathcal{G}(\mathbb{F}, \mu)\right\}=\left\{{ }^{\top} H \cdot M: H \text { is M-integrable }\right\} .
$$

Proof. For $g \in \mathcal{G}(\mathbb{F}, \mu)$, as in the preceding proof, we compute the jumps at one of the $\mathbb{F}$ predictable stopping times $T_{n}<\infty(1 \leq n<\mathrm{N})$.

$$
\Delta_{T_{n}}\left(g_{*}(\mu-\nu)\right)=\sum_{h=1}^{\mathrm{n}} g\left(T_{n}, \alpha_{h, T_{n}}\right) \mathbb{1}_{\left\{\alpha_{h, T_{n}} \neq 0\right\}}\left(\mathbb{1}_{A_{n, h}}-\mathbb{E}\left[\mathbb{1}_{A_{n, h}} \mid \mathcal{F}_{T_{n}-}\right]\right) .
$$

Note that

$$
\left(\mathbb{1}_{A_{n, h}}-\mathbb{E}\left[\mathbb{1}_{A_{n, h}} \mid \mathcal{F}_{T_{n}-}\right]\right)=\left(\mathbb{1}_{A_{n, h}}-p_{n, h}\right)=\sum_{k=1}^{\mathrm{n}}\left(\delta_{h, k}-p_{n, h}\right) \mathbb{1}_{A_{n, k}} .
$$

Taking the conditioning with respect to $\mathcal{F}_{T_{n}-}$, we see

$$
\sum_{k=1}^{\mathrm{n}}\left(\delta_{h, k}-p_{n, h}\right) p_{n, k}=0,
$$

i.e., the vector of components $\left(\delta_{h, k}-p_{n, h}\right)_{1 \leq k \leq \mathrm{n}}$ is orthogonal to $p_{n}$ so that there exists a $\mathcal{F}_{T_{n}-}$-measurable vector $K_{n, h}=\left(K_{n, i, h}\right)_{1 \leq i \leq d}$ such that

$$
\left(\delta_{h, k}-p_{n, h}\right)_{1 \leq k \leq \mathrm{n}}=\sum_{i=1}^{d} \gamma_{n, i} K_{n, i, h},
$$

or in other words, the image in $\mathfrak{L}_{n}$ of the vector $\left(\delta_{h, k}-p_{n, h}\right)_{1 \leq k \leq \mathrm{n}}$ is a combination of the $\Delta_{U_{n}} M_{i}$ :

$$
\begin{aligned}
& \left(\mathbb{1}_{A_{n, h}}-\mathbb{E}\left[\mathbb{1}_{A_{n, h}} \mid \mathcal{F}_{T_{n}-}\right]\right)=\sum_{k=1}^{\mathrm{n}}\left(\delta_{h, k}-p_{n, h}\right) \mathbb{1}_{A_{n, k}} \\
= & \sum_{k=1}^{\mathrm{n}}\left(\sum_{i=1}^{d} \gamma_{n, i} K_{n, i, h}\right)_{k} \mathbb{1}_{A_{n, k}}=\sum_{k=1}^{\mathrm{n}} \sum_{i=1}^{d} \alpha_{n, i, k} K_{n, i, h} \mathbb{1}_{A_{n, k}} \\
= & \sum_{i=1}^{d} K_{n, i, h} \sum_{k=1}^{\mathrm{n}} \alpha_{n, i, k} \mathbb{1}_{A_{n, k}}=\sum_{i=1}^{d} K_{n, i, h} \Delta_{T_{n}} M_{i}={ }^{\top} K_{n, h} \Delta_{T_{n}} M .
\end{aligned}
$$

Hence,

$$
\begin{aligned}
\Delta_{T_{n}}\left(g_{*}(\mu-\nu)\right) & =\sum_{h=1}^{\mathrm{n}} g\left(T_{n}, \alpha_{h, T_{n}}\right) \mathbb{1}_{\left\{\alpha_{h, T_{n}} \neq 0\right\}}\left(\mathbb{1}_{A_{n, h}}-\mathbb{E}\left[\mathbb{1}_{A_{n, h}} \mid \mathcal{F}_{T_{n}-}\right]\right) \\
& =\sum_{h=1}^{\mathrm{n}} g\left(T_{n}, \alpha_{h, T_{n}}\right) \mathbb{1}_{\left\{\alpha_{h, T_{n}} \neq 0\right\}} K_{n, h} \Delta_{T_{n}} M .
\end{aligned}
$$

Set $K_{n}$ to be the matrix $\left(K_{n, i, h}\right)_{1 \leq i \leq d, 1 \leq h \leq \mathrm{n}}$ and $K=\sum_{n=1}^{\mathrm{N}-} K_{n} \mathbb{1}_{\left[T_{n}\right]}$. The above jump identity implies that $K g(\cdot, \alpha) \mathbb{1}_{\{\alpha \neq 0\}}$ is $M$-integrable, and, by [10, Theorem 7.23],

$$
g_{*}(\mu-\nu)={ }^{\top}\left(K g(\cdot, \alpha) \mathbb{1}_{\{\alpha \neq 0\}}\right) \cdot M .
$$

To finish the proof, we recall that $M_{h}=x_{h *}(\mu-\nu)$. 


\subsection{Case of totally inaccessible support}

Consider always an integer valued random measure $\mu$ with its compensator $\nu$, satisfying the finite predictable constraint condition with constraint processes $\alpha_{k}, 1 \leq k \leq \mathrm{n}$, satisfying identity (2). Suppose that $\mathrm{D}=\{\beta \neq$ $\mathbf{0}\}=\cup_{1 \leq n<\mathrm{N}}\left[S_{n}\right]$, where $S_{n}$ are mutually avoiding $\mathbb{F}$ totally inaccessible stopping times and $\mathrm{N}$ a finite or infinite integer.

Suppose in addition that, for a $d$-dimensional $\mathbb{F}$ purely discontinuous local martingale $M, \mathrm{D}=\{\Delta M \neq \mathbf{0}\}$ and $\beta=\Delta M$. ( $M$ is then quasi-left continuous. See [10, Theorem 4.23]). The processes $\alpha_{k}$ are therefore $d$-dimensional vectors. Let $\alpha_{i, k}$ denote the $i$ th component of $\alpha_{k}$ for $1 \leq i \leq d$. As in the preceding paragraph, we define the vector $\gamma_{i}, 1 \leq i \leq d$, to be the vector of the components $\left(\alpha_{i, k}\right)_{1 \leq k \leq \mathrm{n}}$. Denote by $\gamma$ the matrix of columns $\gamma_{i}$ 's.

Theorem 3.8. Let $M$ be a d-dimensional $\mathbb{F}$ purely discontinuous local martingale such that $\mathrm{D}=\{\Delta M \neq \mathbf{0}\}=$ $\cup_{1 \leq n<\mathrm{N}}\left[S_{n}\right]$ and $\beta=\Delta M$ satisfy identity (2). Suppose that, for any $S_{n}$, the vectors $\gamma_{i, S_{n}}, 1 \leq i \leq d$, span the whole space $\mathbb{R}^{\mathrm{n}}$ (so that $\mathrm{n} \leq d$ ). Then, for any $g \in \mathcal{G}(\mathbb{F}, \mu)$, there exists a matrix valued $\mathbb{F}$ predictable process $K$ such that $K g(\cdot, \alpha) \mathbb{1}_{\{\alpha \neq 0\}}$ is $M$-integrable and

$$
g_{*}(\mu-\nu)={ }^{\top}\left(K g(\cdot, \alpha) \mathbb{1}_{\{\alpha \neq 0\}}\right) \cdot M \text {. }
$$

We have the identity

$$
\left\{g_{*}(\mu-\nu): g \in \mathcal{G}(\mathbb{F}, \mu)\right\}=\left\{{ }^{\top} H \cdot M: H \text { is } M \text {-integrable }\right\} .
$$

Proof. Consider the canonical basis $\left(\epsilon_{1}, \ldots, \epsilon_{\mathrm{n}}\right)$ in $\mathbb{R}^{\mathrm{n}}$. Note that the set

$$
\mathbf{A}=\left\{\gamma_{i}, 1 \leq i \leq d, \text { span the whole space } \mathbb{R}^{\mathrm{n}}\right\}
$$

is $\mathbb{F}$ predictable. There exists an $\mathbb{F}$ predictable $d \times n$-matrix valued process $K$ such that

$$
\left(\epsilon_{1}, \ldots, \epsilon_{\mathrm{n}}\right)=\gamma K \text { on } \mathrm{A} .
$$

For any element $g \in \mathcal{G}(\mathbb{F}, \mu)$, we compute the jump at a stopping time $S_{n}<\infty$.

$$
\Delta_{S_{n}}\left(g_{*}(\mu-\nu)\right)=g\left(S_{n}, \beta_{S_{n}}\right)=\sum_{h=1}^{n} g\left(S_{n}, \alpha_{h, S_{n}}\right) \mathbb{1}_{\left\{\alpha_{h, S_{n}} \neq \mathbf{0}\right\}} \mathbb{1}_{\left\{\beta_{S_{n}}=\alpha_{h, S_{n}}\right\}} .
$$

Note (as in the preceding proof) that

$$
\begin{aligned}
& \mathbb{1}_{\left\{\beta_{S_{n}}=\alpha_{h} S_{n}\right\}}=\sum_{k=1}^{\mathrm{n}} \delta_{h, k} \mathbb{1}_{\left\{\beta_{S_{n}}=\alpha_{k, S_{n}}\right\}}=\sum_{k=1}^{\mathrm{n}} \sum_{i=1}^{d} \gamma_{i, k, S_{n}} K_{i, h, S_{n}} \mathbb{1}_{\left\{\beta_{S_{n}}=\alpha_{k, S_{n}}\right\}} \\
= & \sum_{k=1}^{\mathrm{n}} \sum_{i=1}^{d} \alpha_{i, k, S_{n}} K_{i, h, S_{n}} \mathbb{1}_{\left\{\beta_{S_{n}}=\alpha_{k, S_{n}}\right\}}=\sum_{i=1}^{d} K_{i, h, S_{n}} \sum_{k=1}^{\mathrm{n}} \alpha_{i, k, S_{n}} \mathbb{1}_{\left\{\beta_{S_{n}}=\alpha_{k, S_{n}}\right\}} \\
= & \sum_{i=1}^{d} K_{i, h, S_{n}} \Delta_{S_{n}} M_{i} .
\end{aligned}
$$

We conclude

$$
\begin{aligned}
& \Delta_{S_{n}}\left(g_{*}(\mu-\nu)\right)=\sum_{h=1}^{\mathrm{n}} g\left(S_{n}, \alpha_{h, S_{n}}\right) \mathbb{1}_{\left\{\alpha_{h, S_{n}} \neq \mathbf{0}\right\}} \sum_{i=1}^{d} K_{i, h, S_{n}} \Delta_{S_{n}} M_{i} \\
= & { }^{\top}\left(g(\cdot, \alpha) \mathbb{1}_{\{\alpha \neq 0\}}\right){ }_{S_{n}}{ }^{\top} K_{S_{n}} \Delta_{S_{n}} M .
\end{aligned}
$$

This jump identity implies that $K g(\cdot, \alpha) \mathbb{1}_{\{\alpha \neq 0\}}$ is $M$-integrable, and, by [10, Theorem 7.23],

$$
g_{*}(\mu-\nu)={ }^{\top}\left(K g(\cdot, \alpha) \mathbb{1}_{\{\alpha \neq 0\}}\right) \cdot M .
$$




\subsection{Martingale projection property}

Our study on the finite predictable constraint condition has been motivated by the following martingale projection property, which is essential for the proof of Theorem 5.6 and for the proof of [20, Theorem 4.3].

When we compute the predictable bracket $[Y, M]^{\mathbb{F} \cdot p}$ for two local martingales $M, Y$ ( $M$ being, say, locally bounded), we may need to substitute $Y$ by its "orthogonal" projection onto the stable space generated by $M$ :

$$
[Y, M]^{\mathbb{F} \cdot p}=[H \cdot M, M]^{\mathbb{F} \cdot p}
$$

with a stochastic integral $H \cdot M$ with respect to $M$. It is however not always possible, as explained in [3]. On the other hand, as a consequence of [11, Theorem (3.75)], we have a general projection formula for stochastic *-integrals.

Lemma 3.9. Let $M$ be a multiple dimensional purely discontinuous $(\mathbb{P}, \mathbb{F})$ local martingale. Let $\mu$ be its jump measure with $(\mathbb{P}, \mathbb{F})$ compensator $\nu$. For any real $(\mathbb{P}, \mathbb{F})$ local martingale $Y$ such that $[Y, M]$ is $(\mathbb{P}, \mathbb{F})$ locally integrable. There exists a $g \in \mathcal{G}(\mathbb{F}, \mu)$ such that $\left[g_{*}(\mu-\nu), M\right]$ is locally integrable and

$$
[Y, M]^{\mathbb{F} \cdot p}=\left[g_{*}(\mu-\nu), M\right]^{\mathbb{F} \cdot p} .
$$

Proof. Denote by $\mathrm{M}$ the Dolean-Dade measure associated with $\mu$ (cf. [11, Chapitre I (1.32)] for definition). Let $\left(U_{n}\right)_{n \in \mathbb{N}}$ be a sequence of $\mathbb{F}$ stopping times, tending to the infinity, such that $\mathbb{E}\left[\int_{0}^{U_{n}}\left|\mathrm{~d}\left[Y, M_{h}\right]\right|\right]<\infty$ for every component $M_{h}$ of $M$ and for every $n \in \mathbb{N}$. This implies

$$
\mathrm{M}\left[|\Delta Y|\left|x_{h}\right| \mathbb{1}_{\left[0, U_{n}\right]}\right]=\mathbb{E}\left[\sum_{0<s \leq U_{n}}\left|\Delta_{s} Y \Delta_{s} M_{h}\right| \mathbb{1}_{\left\{\Delta_{s} M \neq 0\right\}}\right]=\mathbb{E}\left[\int_{0}^{U_{n}}\left|\mathrm{~d}\left[Y, M_{h}\right]\right|\right]<\infty .
$$

With the notations in $[11$, Theorem (3.75)] let

$$
U=\mathrm{M}[\Delta Y \mid \widetilde{\mathcal{P}}], g=U+\frac{\widehat{U}}{1-a}, V=\Delta Y-U .
$$

Then, $g \in \mathcal{G}(\mathbb{F}, \mu)$ and

$$
Y=g_{*}(\mu-\nu)+V_{*} \mu+Y^{\prime},
$$

where $Y^{\prime}$ is a local martingale pathwise orthogonal to $M$, i.e. $\left[Y^{\prime}, M\right] \equiv 0$. Consider $\left[V_{*} \mu, M_{h}\right]$. We have

$$
\begin{aligned}
& \mathbb{E}\left[\int_{0}^{U_{n}}\left|\mathrm{~d}\left[V_{*} \mu, M_{h}\right]\right|\right]=\mathbb{E}\left[\sum_{0<s \leq U_{n}}\left|\left(\Delta_{s} Y-U\left(s, \Delta_{s} M\right)\right) \Delta_{s} M_{h}\right| \mathbb{1}_{\left\{\Delta_{s} M \neq 0\right\}}\right] \\
\leq & \mathbb{E}\left[\sum_{0<s \leq U_{n}}\left|\Delta_{s} Y \Delta_{s} M_{h}\right| \mathbb{1}_{\left\{\Delta_{s} M \neq 0\right\}}\right]+\mathbb{E}\left[\sum_{0<s \leq U_{n}}\left|U\left(s, \Delta_{s} M\right) \Delta_{s} M_{h}\right| \mathbb{1}_{\left\{\Delta_{s} M \neq 0\right\}}\right] \\
= & \mathrm{M}\left[|\Delta Y|\left|x_{h}\right| \mathbb{1}_{\left[0, U_{n}\right]}\right]+\mathrm{M}\left[|U|\left|x_{h}\right| \mathbb{1}_{\left[0, U_{n}\right]}\right] \\
\leq & 2 \mathrm{M}\left[|\Delta Y|\left|x_{h}\right| \mathbb{1}_{\left[0, U_{n}\right]}\right]<\infty .
\end{aligned}
$$

This means that $\left[V_{*} \mu, M_{h}\right]^{\mathbb{F} \cdot p}$ is defined. But for any $\mathbb{F}$ stopping time $S$,

$$
\mathbb{E}\left[\left[V_{*} \mu, M_{h}\right]_{S \wedge U_{n}}\right]=\mathrm{M}\left[(\Delta Y-U) x_{h} \mathbb{1}_{\left[0, S \wedge U_{n}\right]}\right]=0,
$$

i.e. $\left[V_{*} \mu, M_{h}\right]^{\mathbb{F} \cdot p}=0$. As $\left[Y, M_{h}\right]^{\mathbb{F} \cdot p}$ exists, necessarily $\left[g_{*}(\mu-\nu), M_{h}\right]^{\mathbb{F} \cdot p}$ exists and

$$
[Y, M]^{\mathbb{F} \cdot p}=\left[g_{*}(\mu-\nu), M\right]^{\mathbb{F} \cdot p} .
$$

Remark 3.10. In the light of Lemma 3.9, we understand that, to have the martingale projection property (4), it is enough to transform the stochastic ${ }_{*}$-integrals $g_{*}(\mu-\nu)$ into stochastic --integrals $H \cdot M$, i.e., to employ Theorem 3.7 and Theorem 3.8. This remark is applied in Section 5. 


\section{Martingale Representation PRoperty}

Fix a stochastic basis $(\Omega, \mathbb{F}, \mathbb{P})$.

Definition 4.1. We say that the martingale representation property holds in the filtration $\mathbb{F}$ (under the probability $\mathbb{P}$ ) with respect to a d-dimensional representation process $W$, if $W$ is a $(\mathbb{P}, \mathbb{F})$ local martingale, and every $(\mathbb{P}, \mathbb{F})$ local martingale is a stochastic integral with respect to $W$. We also say that $W$ possesses the martingale representation property in $\mathbb{F}$. We say simply that the martingale representation property holds in $\mathbb{F}$, if there exists some finite dimensional $(\mathbb{P}, \mathbb{F})$ local martingale which possesses the martingale representation property in $\mathbb{F}$.

As mentioned in Introduction, in the present paper, the martingale representation property is considered as a property of the reference filtration $\mathbb{F}$, rather than the property of a specific $\mathbb{F}$ local martingale. Actually, the representation process is not unique. When we make computations under the martingale representation property, we are not restricted to work with the initially given representation process $W$. In choosing suitable representation process, we can render the computations much easier. This reflection on the representation process was the consequence of the attempt to improve the paper [19]. That paper was the first study on the full viability in a non classic setting. A (fairly restrictive) sufficient condition had been proved. It was not satisfactory, because what mattered was rather the consequence of the full viability. In changing the representation process (cf. Theorem 4.2 below), we had succeeded to extend the sufficient condition in [19] to the necessary and sufficient condition in [20].

Theorem 4.2. If the martingale representation property holds in $\mathbb{F}$ under $\mathbb{P}$, there exists always a locally bounded representation process, which has pathwise orthogonal components outside of a predictable thin set. Precisely, there exist a continuous local martingale $Z^{\prime}$ and two locally bounded purely discontinuous local martingales $Z^{\prime \prime}, Z^{\prime \prime \prime}$ with respectively only accessible jumping times or totally inaccessible jumping times, such that $Z=$ $\left(Z^{\prime}, Z^{\prime \prime}, Z^{\prime \prime \prime}\right)$ forms a representation process. Moreover, the path of $Z^{\prime \prime}$ is of finite variation and, in the multidimensional case, the components of the processes $Z^{\prime}, Z^{\prime \prime \prime}$ are mutually pathwise orthogonal.

This section is devoted to the proof of Theorem 4.2 .

\subsection{Conditional multiplicity}

The martingale representation property imposes, for any $\mathbb{F}$ stopping time $R$, the finite conditional multiplicity of $\mathcal{F}_{R}$ with respect to $\mathcal{F}_{R-}$ (a notion introduced in [5, section 3] to quantify the randomness of $\mathcal{F}_{R}$ when $\mathcal{F}_{R-}$ is given).

Lemma 4.3. Suppose the martingale representation property in $\mathbb{F}$ with a d-dimensional representation process $W$. Let $R$ be an $\mathbb{F}$ stopping time. Consider the random variables in $\mathcal{F}_{R-}$ as constants. If $R$ is predictable, the family of random variables $\Delta_{R} W_{h}, 1 \leq h \leq d$, generates on $\{R<\infty\}$ (modulo $\mathcal{F}_{R-}$ ) all integrable random variables $\xi$ in $\mathcal{F}_{R}$ whose conditional expectation $\mathbb{E}\left[\xi \mid \mathcal{F}_{R-}\right]=0$. If $R$ is totally inaccessible, the family of $\Delta_{R} W_{h}, 1 \leq h \leq d$ generates on $\{R<\infty\}$ (modulo $\mathcal{F}_{R-}$ ) all integrable random variables $\xi$ in $\mathcal{F}_{R}$.

Proof. For any integrable $\xi \in \mathcal{F}_{R}$, the process $\xi \mathbb{1}_{[R, \infty)}-\left(\xi \mathbb{1}_{[R, \infty)}\right)^{\mathbb{F} \cdot p}$ is a martingale. By martingale representation property, there exists an $\mathbb{F}$-predictable process $H$ such that $\xi \mathbb{1}_{[R, \infty)}-\left(\xi \mathbb{1}_{[R, \infty)}\right)^{\mathbb{F} \cdot p}={ }^{\top} H \cdot W$. Therefore,

$$
\xi=\sum_{h=1}^{d}\left(H_{R}\right)_{h} \Delta_{R} W_{h}+\Delta_{R}\left(\xi \mathbb{1}_{[R, \infty)}\right)^{\mathbb{F} \cdot p}
$$

on $\{R<\infty\}$. If $R$ is predictable and $\mathbb{E}\left[\xi \mid \mathcal{F}_{R-}\right]=0$, the process $\left(\xi \mathbb{1}_{[R, \infty)}\right)^{\mathbb{F} \cdot p}=0$. If $R$ is totally inaccessible, $\Delta_{R}\left(\xi \mathbb{1}_{[R, \infty)}\right)^{\mathbb{F} \cdot p}=0$. The lemma is proved. 
Lemma 4.4. Suppose the martingale representation property in $\mathbb{F}$ with a d-dimensional representation process $W$. If $R$ is $\mathbb{F}$ predictable, there exists a partition $\left(A_{0}, A_{1}, A_{2}, \ldots, A_{d}\right)$ (where some $A_{i}$ may be empty) such that

$$
\mathcal{F}_{R}=\mathcal{F}_{R-} \vee \sigma\left(A_{0}, A_{1}, A_{2}, \ldots, A_{d}\right)
$$

i.e. the conditional multiplicity of $\mathcal{F}_{R}$ with respect to $\mathcal{F}_{R-}$ is equal to or smaller then $d+1$. If $R$ is $(\mathbb{P}, \mathbb{F})$ totally inaccessible, there exists a partition $\left(B_{1}, B_{2}, \ldots, B_{d}\right)$ (where some $B_{j}$ may be empty) such that

$$
\mathcal{F}_{R}=\mathcal{F}_{R-} \vee \sigma\left(B_{1}, B_{2}, \ldots, B_{d}\right)
$$

i.e. the conditional multiplicity of $\mathcal{F}_{R}$ with respect to $\mathcal{F}_{R-}$ is equal to or smaller then $d$.

Proof. Consider the case of a predictable $R$. Because of Lemma 4.3, we can apply [5, Proposition 12] to have a partition $\left(A_{0}^{\prime}, A_{1}^{\prime}, A_{2}^{\prime}, \ldots, A_{d}^{\prime}\right)$ of $\{R<\infty\}$ such that

$$
\{R<\infty\} \cap \mathcal{F}_{R}=\{R<\infty\} \cap\left(\mathcal{F}_{R-} \vee \sigma\left(A_{0}^{\prime}, A_{1}^{\prime}, A_{2}^{\prime}, \ldots, A_{d}^{\prime}\right)\right) .
$$

Since $\{R=\infty\} \cap \mathcal{F}_{R}=\{R=\infty\} \cap \mathcal{F}_{R-}$, the lemma is verified, if we take $A_{i}=A_{i}^{\prime}$ for $0 \leq i<d$ and $A_{d}=A_{d}^{\prime} \cup\{R=\infty\}$.

The case of a totally inaccessible $R$ can be dealt with similarly.

\subsection{A separation technique}

Suppose the martingale representation property in $\mathbb{F}$ with a representation process $W$. Then, the $(\mathbb{P}, \mathbb{F})$ local martingale $X$ takes all the form ${ }^{\top} H \cdot W$ for some $W$-integrable predictable process $H$. We call (any version of) the process $H$ the coefficient of $X$ in its martingale representation with respect to the process $W$. This appellation extends naturally to vector valued local martingales.

When we compute a purely discontinuous local martingale $X$ with its martingale representation, we may like to decompose its representation coefficient $H$ into parts $\sum_{k \geq 1} H_{k}$ such that, for each $k, H_{k, R}=H_{R}$ at a specific jumping time $R$ of $X$ and $H_{k, R^{\prime}}=0$ at the other jumping times $R^{\prime}$ (so that $H_{k}$ carries all, and only, the information about the jump $\Delta_{R} X$ ). This decomposition is possible if all the jumping times are predictable. In a general case, the following lemma can be useful.

Lemma 4.5. Let $R$ be any $\mathbb{F}$ stopping time. Let $\xi \in \mathbf{L}^{1}\left(\mathbb{P}, \mathcal{F}_{R}\right)$. Let $H$ denote any coefficient of the $(\mathbb{P}, \mathbb{F})$ martingale $\xi \mathbb{1}_{[R, \infty)}-\left(\xi \mathbb{1}_{[R, \infty)}\right)^{\mathbb{F} \cdot p}$ in its martingale representation with respect to $W$.

(1) If $R$ is predictable, the two predictable processes $H$ and $H \mathbb{1}_{[R]}$ are in the same equivalence class with respect to $W$, whose value is determined by the equation on $\{R<\infty\}$

$$
{ }^{\top} H_{R} \Delta_{R} W=\xi-\mathbb{E}\left[\xi \mid \mathcal{F}_{R-}\right] .
$$

(2) If $R$ is totally inaccessible, the process $H$ satisfies the equations on $\{R<\infty\}$

$$
{ }^{\top} H_{R} \Delta_{R} W=\xi, \text { and }{ }^{\top} H_{S} \Delta_{S} W=0 \text { on }\{S \neq R\},
$$

for any $\mathbb{F}$ stopping time $S$.

Proof. Let us consider only a totally inaccessible stopping time $R$. In this case, $\left(\xi \mathbb{1}_{[R, \infty)}\right)^{\mathbb{F} \cdot p}$ is continuous. Computing the jump at $R$ and at $S$ in the equation

$$
\xi \mathbb{1}_{[R, \infty)}-\left(\xi \mathbb{1}_{[R, \infty)}\right)^{\mathbb{F} \cdot p}={ }^{\top} H \cdot W
$$

we prove the assertions. 


\subsection{Representation process reconstituted}

Definition 4.6. For a multi-dimensional $\mathbb{F}$ local martingale $X$, we say that it has pathwise orthogonal components, if $\left[X_{i}, X_{j}\right]=0$ for $i \neq j$. For a measurable set $\mathrm{A}$, we say that $X$ has pathwise orthogonal components outside of $\mathrm{A}$, if $\mathbb{1}_{\mathrm{A}^{c}} \cdot\left[X_{i}, X_{j}\right]=0$ for $i \neq j$.

Suppose in the rest of this section that the martingale representation property holds in $\mathbb{F}$ with a $d$-dimensional representation process $W$. The following lemma is well-known (cf. [7]).

Lemma 4.7. There exists a continuous d-dimensional $\mathbb{F}$ local martingale $X^{\prime}$ which generates the same stable space as that generated by the components of $W^{c}$, but with pathwise orthogonal components (some of the components may be identically null).

Consider the purely discontinuous part $W^{d}$. We introduce the following notations. Let $\left(S_{n}\right)_{1 \leq n<\mathrm{N}^{i}}\left(\mathrm{~N}^{i} \leq \infty\right)$ (resp. $\left(T_{n}\right)_{1 \leq n<\mathrm{N}^{a}}$ ) be a sequence of $(\mathbb{P}, \mathbb{F})$ totally inaccessible (resp. strictly positive $(\mathbb{P}, \mathbb{F})$ predictable) stopping times such that $\left[S_{n}\right] \cap\left[S_{n^{\prime}}\right]=\emptyset$ for $n \neq n^{\prime}$ and $\left\{s \geq 0: \Delta_{s} W^{d i} \neq 0\right\} \subset \cup_{n \geq 1}\left[S_{n}\right]$ (resp. $\left[T_{n}\right] \cap\left[T_{n^{\prime}}\right]=\emptyset$ for $n \neq n^{\prime}$ and $\left.\left\{s \geq 0: \Delta_{s} W^{d a} \neq 0\right\} \subset \cup_{n \geq 1}\left[T_{n}\right]\right)$.

For $1 \leq n^{\prime}<\mathrm{N}^{a}$, for $1 \leq n<\mathrm{N}^{i}$, we find and enumerate the partition sets defined in Lemma 4.4 for the stopping times $T_{n^{\prime}}$ or $S_{n}:\left(A_{n^{\prime}, 0}, A_{n^{\prime}, 1}, A_{n^{\prime}, 2}, \ldots, A_{n^{\prime}, d}\right)$ and $\left(B_{n, 1}, B_{n, 2}, \ldots, B_{n, d}\right)$ (where some $A_{h^{\prime}}$ and $B_{h}$ may be empty) such that

$$
\mathcal{F}_{T_{n^{\prime}}}=\mathcal{F}_{T_{n^{\prime}}} \vee \vee \sigma\left(A_{n^{\prime}, 0}, A_{n^{\prime}, 1}, A_{n^{\prime}, 2}, \ldots, A_{n^{\prime}, d}\right) \text { and } \mathcal{F}_{S_{n}}=\mathcal{F}_{S_{n}-} \vee \sigma\left(B_{n, 1}, B_{n, 2}, \ldots, B_{n, d}\right) .
$$

Let

$$
p_{n^{\prime}, h^{\prime}}=\mathbb{P}\left[A_{n^{\prime}, h^{\prime}} \mid \mathcal{F}_{T_{n^{\prime}}-}\right] \quad \text { and } q_{n, h}=\mathbb{P}\left[B_{n, h} \mid \mathcal{F}_{S_{n}-}\right], 0 \leq h^{\prime} \leq d, 1 \leq h \leq d
$$

Let

$$
v_{n^{\prime}, h^{\prime}} \in \mathcal{F}_{T_{n}-} \text { and respectively } w_{n, h} \in \mathcal{F}_{S_{n}-}
$$

be the vector value of $\Delta_{T_{n^{\prime}}} W$ on $A_{n^{\prime}, h^{\prime}}$ and respectively the vector value of $\Delta_{S_{n}} W$ on $B_{n, h}$ (cf. Lemma 4.4 ). We define real processes

$$
X_{h^{\prime}}^{\prime \prime}=\sum_{1 \leq n^{\prime}<\mathrm{N}^{a}} \frac{1}{2^{n}}\left(\mathbb{1}_{A_{n^{\prime}, h^{\prime}}} \mathbb{1}_{\left[T_{n^{\prime}}, \infty\right)}-\left(\mathbb{1}_{A_{n^{\prime}, h^{\prime}}} \mathbb{1}_{\left[T_{n^{\prime}}, \infty\right)}\right)^{\mathbb{F} \cdot p}\right), 0 \leq h^{\prime} \leq d
$$

We define $d$-dimensional vector valued processes

$$
X_{h}^{\prime \prime \prime}=\sum_{1 \leq n<\mathbb{N}^{i}} \frac{1}{2^{n}}\left(w_{n, h} \mathbb{1}_{B_{n, h}} \mathbb{1}_{\left[S_{n}, \infty\right)}-\left(w_{n, h} \mathbb{1}_{B_{n, h}} \mathbb{1}_{\left[S_{n}, \infty\right)}\right)^{\mathbb{F} \cdot p}\right), 1 \leq h \leq d,
$$

(which is well-defined). Let $X=\left(X^{\prime}, X^{\prime \prime}, X^{\prime \prime \prime}\right)$, i.e., the multi-dimensional local martingale whose components are composed of the components of respectively $X^{\prime}, X^{\prime \prime}, X^{\prime \prime \prime}$ in their natural order.

Theorem 4.8. The process $X$ has the martingale representation property in $\mathbb{F}$ under $\mathbb{P}$.

Proof. Let $Y$ be a $($ real $)$ bounded $(\mathbb{P}, \mathbb{F})$ martingale such that the bracket $[Y, X]$ is a vector valued $(\mathbb{P}, \mathbb{F})$ local martingales (i.e., $Y$ is orthogonal to the components of $X$ in the local martingale sense, cf. [3, 11]). By the martingale representation property of $W$ in $(\mathbb{P}, \mathbb{F}), Y$ takes the form $Y={ }^{\top} H \cdot W$ for some vector valued $\mathbb{F}$ predictable process $H$. The computation of the bracket gives

$$
\begin{aligned}
{\left[Y, X_{h}^{\prime \prime}\right] } & =\sum_{1 \leq n<\mathrm{N}^{a}} \frac{1}{2^{n}}\left[Y, \mathbb{1}_{A_{n, h}} \mathbb{1}_{\left[T_{n}, \infty\right)}-\left(\mathbb{1}_{A_{n, h},} \mathbb{1}_{\left[T_{n}, \infty\right)}\right)^{\mathbb{F} \cdot p]}\right. \\
& =\sum_{1 \leq n<\mathrm{N}^{a}} \frac{1}{2^{n}} H \cdot\left[W, \mathbb{1}_{A_{n, h}} \mathbb{1}_{\left[T_{n}, \infty\right)}-\left(\mathbb{1}_{A_{n, h}} \mathbb{1}_{\left[T_{n}, \infty\right)}\right)^{\mathbb{F} \cdot p}\right] \\
& =\sum_{1 \leq n<\mathrm{N}^{a}} \frac{1}{2^{n} \top} H_{T_{n}} \Delta_{T_{n}} W\left(\mathbb{1}_{A_{n, h}}-p_{n, h}\right) \mathbb{1}_{\left[T_{n}, \infty\right)} \\
& =\sum_{1 \leq n<\mathrm{N}^{a}} \frac{1}{2^{n} \top} H_{T_{n}} \Delta_{T_{n}} W \mathbb{1}_{A_{n, h}} \mathbb{1}_{\left[T_{n}, \infty\right)}-\sum_{1 \leq n<\mathrm{N}^{a}} \frac{1}{2^{n}}{ }^{\top} H_{T_{n}} \Delta_{T_{n}} W p_{n, h} \mathbb{1}_{\left[T_{n}, \infty\right)} \\
& =\sum_{1 \leq n<\mathrm{N}^{a}} \frac{1}{2^{n}}{ }^{\top} H_{T_{n}} v_{n, h} \mathbb{1}_{A_{n, h}} \mathbb{1}_{\left[T_{n}, \infty\right)}-\sum_{1 \leq n<\mathrm{N}^{a}} \frac{1}{2^{n}}{ }^{\top} H_{T_{n}} \Delta_{T_{n}} W p_{n, h} \mathbb{1}_{\left[T_{n}, \infty\right)} .
\end{aligned}
$$


It is a $(\mathbb{P}, \mathbb{F})$ local martingale. For every $1 \leq n<\mathrm{N}^{a}$, taking the stochastic integral of the predictable process $\mathbb{1}_{\left[T_{n}\right]}$ with respect to this local martingale, we see that each term

$$
\frac{1}{2^{n}}{ }^{\top} H_{T_{n}} v_{n, h} \mathbb{1}_{A_{n, h}} \mathbb{1}_{\left[T_{n}, \infty\right)}-\frac{1}{2^{n}}{ }^{\top} H_{T_{n}} \Delta_{T_{n}} W p_{n, h} \mathbb{1}_{\left[T_{n}, \infty\right)}
$$

is itself a local martingale. Taking the predictable dual projection, we obtain

$$
\frac{1}{2^{n}}{ }^{\top} H_{T_{n}} v_{n, h} p_{n, h} \mathbb{1}_{\left[T_{n}, \infty\right)} \equiv 0 \text { (a null process) }
$$

because $\mathbb{E}\left[{ }^{\top} H_{T_{n}} \Delta_{T_{n}} W \mid \mathcal{F}_{T_{n}}\right]=0$ and $v_{n, h} \in \mathcal{F}_{T_{n}-}$. Consequently ${ }^{\top} H_{T_{n}} v_{n, h} \mathbb{1}_{A_{n, h}}=0$ on $\left\{T_{n}<\infty\right\}$. This being true for any $0 \leq h \leq d$, we can write

$$
\begin{aligned}
\Delta_{T_{n}} Y & ={ }^{\top} H_{T_{n}} \Delta_{T_{n}} W=\sum_{h=0}^{d}{ }^{\top} H_{T_{n}} \Delta_{T_{n}} W \mathbb{1}_{A_{n, h}} \\
& =\sum_{h=0}^{d}{ }^{\top} H_{T_{n}} v_{n, h} \mathbb{1}_{A_{n, h}}=0, \quad \text { on }\left\{T_{n}<\infty\right\} .
\end{aligned}
$$

In the same way,

$$
\begin{aligned}
{\left[Y, X_{h}^{\prime \prime \prime}\right] } & =\sum_{1 \leq n<\mathrm{N}^{i}} \frac{1}{2^{n}}\left[Y, w_{n, h} \mathbb{1}_{B_{n, h}} \mathbb{1}_{\left[S_{n}, \infty\right)}-\left(w_{n, h} \mathbb{1}_{B_{n, h}} \mathbb{1}_{\left[S_{n}, \infty\right)}\right)^{\mathbb{F} \cdot p]}\right. \\
& =\sum_{1 \leq n<\mathrm{N}^{i}} \frac{1}{2^{n}}\left[Y, w_{n, h} \mathbb{1}_{B_{n, h}} \mathbb{1}_{\left[S_{n}, \infty\right)}\right] \text { because }\left(w_{n, h} \mathbb{1}_{B_{n, h}} \mathbb{1}_{\left[S_{n}, \infty\right)}\right)^{\mathbb{F} \cdot p} \text { is continuous} \\
& =\sum_{1 \leq n<\mathrm{N}^{a}} \frac{w_{n, h}}{2^{n}} H_{S_{n}} \Delta_{S_{n}} W \mathbb{1}_{B_{n, h}} \mathbb{1}_{\left[S_{n}, \infty\right)} \\
& =\sum_{1 \leq n<\mathrm{N}^{a}} \frac{w_{n, h}}{2^{n}} H_{S_{n}} w_{n, h} \mathbb{1}_{B_{n, h}, \mathbb{1}_{\left[S_{n}, \infty\right)}}
\end{aligned}
$$

is a local martingale. For $1 \leq i<\mathbf{N}^{i}$, set $J_{i}$ the coefficient of $\mathbb{1}_{B_{i, h}} \mathbb{1}_{\left[S_{i}, \infty\right)}-\left(\mathbb{1}_{B_{i, h}} \mathbb{1}_{[R, \infty)}\right)^{\mathbb{F} \cdot p}$ in its martingale representation with respect to $W$. By Lemma 4.5,

$$
\begin{aligned}
& { }^{\top} J_{i} \cdot\left(\sum_{1 \leq n<\mathrm{N}^{a}} \frac{w_{n, h}}{2^{n}} H_{S_{n}} w_{n, h} \mathbb{1}_{B_{n, h}} \mathbb{1}_{\left[S_{n}, \infty\right)}\right)=\sum_{1 \leq n<\mathrm{N}^{a}} \frac{{ }^{\top} J_{i, S_{n}} w_{n, h}}{2^{n}}{ }^{\top} H_{S_{n}} w_{n, h} \mathbb{1}_{B_{n, h}} \mathbb{1}_{\left[S_{n}, \infty\right)} \\
= & \frac{1}{2^{i}}{ }^{\top} J_{i, S_{i}} w_{i, h}^{\top} H_{S_{i}} w_{i, h} \mathbb{1}_{B_{i, h}} \mathbb{1}_{\left[S_{i}, \infty\right)}=\frac{1}{2^{i}}{ }^{\top} H_{S_{i}} w_{i, h} \mathbb{1}_{B_{i, h}} \mathbb{1}_{\left[S_{i}, \infty\right)} .
\end{aligned}
$$

By assumption, it is a $(\mathbb{P}, \mathbb{F})$ local martingale. Hence, $\frac{1}{2^{i}}{ }^{\top}\left(H_{S_{i}} w_{n, h} q_{n, h} \mathbb{1}_{\left[S_{n}, \infty\right)}\right)^{\mathbb{F} \cdot p}$ is a null process. Repeating the reasoning in the preceding paragraphs, we conclude that ${ }^{\top} H_{S_{i}} w_{i, h} \mathbb{1}_{B_{i, h}}=0$ so that $\Delta_{S_{i}} Y=0$.

Hence $Y$ is a continuous martingale. As the continuous components $X^{\prime}$ generate $W^{c}$, the bracket ${ }^{\top} H$. $\left\langle W^{c}, W_{k}^{c}\right\rangle, 1 \leq k \leq d$, is a local martingale, which must be null. Consequently $H \cdot W^{c}=0$. This proves the theorem, according to [11, Corollaire(4.12)].

\subsection{The finite predictable constraint condition of representation processes}

Under the martingale representation property, the representation processes satisfy the finite predictable constraint. This can result from [11, Théorème 4.80]. For our account of the martingale representation property to be complete, to follow the logic of the present paper, we give here a brief description of this result, in terms of the processes $X^{\prime \prime}, X^{\prime \prime \prime}$.

Lemma 4.9. There exist a finite number $\mathrm{n}^{\prime \prime}$ of $d+1$-dimensional $\mathbb{F}$ predictable process $\alpha_{k}^{\prime \prime}, 1 \leq k \leq \mathrm{n}^{\prime \prime}$, such that

$$
\Delta X^{\prime \prime} \mathbb{1}_{\left\{\Delta X^{\prime \prime} \neq \mathbf{0}\right\}}=\sum_{k=1}^{\mathrm{n}^{\prime \prime}} \alpha_{k}^{\prime \prime} \mathbb{1}_{\left\{\Delta X^{\prime \prime}=\alpha_{k}^{\prime \prime}\right\}} .
$$

Proof. It is enough to notice that, for every component

$$
X_{h}^{\prime \prime}=\sum_{1 \leq n<\mathrm{N}^{a}} \frac{1}{2^{n}}\left(\mathbb{1}_{A_{n, h}} \mathbb{1}_{\left[T_{n}, \infty\right)}-\left(\mathbb{1}_{A_{n, h}} \mathbb{1}_{\left[T_{n}, \infty\right)}\right)^{\mathbb{F} \cdot p}\right)=\sum_{1 \leq n<\mathrm{N}^{a}} \frac{1}{2^{n}}\left(\mathbb{1}_{A_{n, h}}-p_{n, h}\right) \mathbb{1}_{\left[T_{n}, \infty\right)},
$$


$0 \leq h \leq d$, the jump process $\Delta X_{h}^{\prime \prime}$ takes one of the three values $\mathbf{0}, \zeta_{h, 1}, \zeta_{h, 2}$, where

$$
\zeta_{h, 1}=\sum_{1 \leq n<\mathrm{N}^{a}} \frac{1}{2^{n}}\left(1-p_{n, h}\right) \mathbb{1}_{\left[T_{n}\right]}, \quad \zeta_{h, 2}=\sum_{1 \leq n<\mathrm{N}^{a}} \frac{1}{2^{n}}\left(-p_{n, h}\right) \mathbb{1}_{\left[T_{n}\right]},
$$

which are $\mathbb{F}$ predictable processes.

Lemma 4.10. Suppose that $W$ is locally square integrable. There exist a finite number $\mathrm{n}^{\prime \prime \prime}$ of $d \times d$-dimensional $\mathbb{F}$ predictable process $\alpha_{k}^{\prime \prime \prime}, 1 \leq k \leq \mathrm{n}^{\prime \prime \prime}$, such that

$$
\Delta X^{\prime \prime \prime} \mathbb{1}_{\left\{\Delta X^{\prime \prime \prime} \neq \mathbf{0}\right\}}=\sum_{k=1}^{\mathrm{n}^{\prime \prime \prime}} \alpha_{k}^{\prime \prime \prime} \mathbb{1}_{\left\{\Delta X^{\prime \prime \prime}=\alpha_{k}^{\prime \prime \prime}\right\}} .
$$

Proof. Notice that $X^{\prime \prime \prime}$ is here locally square integrable. For $1 \leq h \leq d$, let $\mu$ be the jump measure of $X_{h}^{\prime \prime \prime}$ with $\mathbb{F}$ compensator $\nu$. We consider, for $1 \leq i, j \leq d$, the local martingale $x_{i} x_{j *}(\mu-\nu)$ and its representation by $X$. By the pathwise orthogonality, its representation depends only on $X_{h}^{\prime \prime \prime}$. Hence, there exists a $\mathbb{F}$ predictable process $H_{j}, 1 \leq j \leq d$, such that

Computing the jumps, we obtain

$$
{ }^{\top} H_{j} \cdot X_{h}^{\prime \prime \prime}=x_{i} x_{j *}(\mu-\nu) .
$$

$$
\sum_{k=1}^{d} H_{j, k} \Delta X_{h, k}^{\prime \prime \prime}=\Delta X_{h, i}^{\prime \prime \prime} \Delta X_{h, j}^{\prime \prime \prime}, 1 \leq j \leq d .
$$

This means that, if $\Delta X_{h}^{\prime \prime \prime} \neq 0, \Delta X_{h, i}^{\prime \prime \prime}$ is a root of the characteristic polynomial of the matrix of components $\left(H_{j, k}\right)_{1 \leq j, k \leq d}$. Applying [4, Theorem 2.2], there exists $\mathbb{F}$ predictable processes $\left(\zeta_{1}, \ldots, \zeta_{d}\right)$ such that

$$
\Delta X_{h, i}^{\prime \prime \prime} \prod_{j=1}^{d}\left(\Delta X_{h, i}^{\prime \prime \prime}-\zeta_{j}\right)=0 .
$$

The lemma can now be deduced from this property.

Theorem 4.11. Suppose that $W$ has the martingale representation property in $\mathbb{F}$ under $\mathbb{P}$. Then, the process $W$ satisfies the finite $\mathbb{F}$ predictable constraint condition. More precisely, there exist a finite number $\mathrm{n}$ of $d$ dimensional $\mathbb{F}$ predictable process $\alpha_{k}, 1 \leq k \leq \mathrm{n}$, such that

$$
\Delta W \mathbb{1}_{\{\Delta W \neq \mathbf{0}\}}=\sum_{k=1}^{\mathrm{n}} \alpha_{k} \mathbb{1}_{\left\{\Delta W=\alpha_{k}\right\}} .
$$

Proof. If $W$ is locally square integrable, the theorem is the consequence of the representation of $W$ by $X$ (Theorem 4.8) and of Lemma 4.9 and Lemma 4.10. If not, let $T>0$ be a constant. Let $W_{T}^{*}=\sup _{s \leq T}\left|W_{s}\right|$. Let $\eta=e^{-W_{T}^{*}}$ and let $\left(\eta_{t}\right)_{t \in[0, T]}$ be the associated $(\mathbb{P}, \mathbb{F})$ bounded martingale. Let $\overline{\mathbb{P}}=\eta \cdot \mathbb{P}$ and

$$
\bar{W}=W-\frac{1}{\eta_{-}} \cdot\langle\eta, W\rangle^{\mathbb{P} \cdot \mathbb{F}} .
$$

The process $\bar{W}$ is locally square integrable under $\overline{\mathbb{P}}$ on the interval $[0, T]$, and by [14], $\bar{W}$ possesses the martingale representation property in $\mathbb{F}$ under $\overline{\mathbb{P}}$. There exist, therefore, a finite number $\mathrm{n}$ (independent of $T$ ) of $\mathbb{F}$ predictable processes $\left(\zeta_{1}, \ldots, \zeta_{\mathrm{n}}\right)$ such that

$$
\Delta \bar{W} \in\left\{\mathbf{0}, \zeta_{1}, \ldots \ldots, \zeta_{\mathrm{n}}\right\}
$$


on $[0, T]$, or equivalently

$$
\Delta W \in\left\{\mathbf{0}, \zeta_{1}+\frac{1}{\eta_{-}} \Delta\langle\eta, W\rangle^{\mathbb{P} \cdot \mathbb{F}}, \ldots \ldots, \zeta_{\mathrm{n}}+\frac{1}{\eta_{-}} \Delta\langle\eta, W\rangle^{\mathbb{P} \cdot \mathbb{F}}\right\}
$$

on $[0, T]$. The theorem is deduced from this property.

Corollary 4.12. Lemma 4.10 remain available, without the local square integrability of $W$.

\subsection{Another modification of the representation process}

The process $X^{\prime \prime \prime}$ is not always locally bounded and has not necessarily pathwise orthogonal components. With the finite predictable constraint condition, we can modify it to have the boundedness and the pathwise orthogonality.

Consider the process $\alpha^{\prime \prime \prime}$ in Lemma 4.10 (cf. Corollary 4.12). Let $\mu$ be the jump measure of $X^{\prime \prime \prime}$ with $\mathbb{F}$ compensator $\nu$. Let $e_{k}, u_{k}, 1 \leq k \leq \mathrm{n}^{\prime \prime \prime}$, be the function in Theorem 3.3 relative to $\alpha^{\prime \prime \prime}$. Let

$$
X_{k}^{\circ}=u_{k *}(\mu-\nu), 1 \leq k \leq \mathrm{n}^{\prime \prime \prime} .
$$

The local martingales $X_{k}^{\circ}$ are mutually pathwise orthogonal.

Lemma 4.13. For any $X^{\prime \prime \prime}$-integrable $\mathbb{F}$ predictable process $H$,

$$
{ }^{\top} H \cdot X^{\prime \prime \prime}={ }^{\top} H \frac{\alpha^{\prime \prime \prime}}{\mathrm{e}\left(\alpha^{\prime \prime \prime}\right)} \mathbb{1}_{\left\{\mathrm{e}\left(\alpha^{\prime \prime \prime}\right) \neq 0\right\}} \cdot X^{\circ} .
$$

Proof. We have, by [10, Theorem 11.23 and 11.24],

$$
{ }^{\top} H \cdot X^{\prime \prime \prime}={ }^{\top} H \cdot\left(x_{*}(\mu-\nu)\right)={ }^{\top} H x_{*}(\mu-\nu) .
$$

Applying Theorem 3.3, we see that ${ }^{\top} H \frac{\alpha^{\prime \prime \prime}}{\mathrm{e}\left(\alpha^{\prime \prime \prime}\right)} \mathbb{1}_{\left\{\mathrm{e}\left(\alpha^{\prime \prime \prime}\right) \neq 0\right\}}$ is $X^{\circ}$-integrable and

$$
{ }^{\top} H \cdot X^{\prime \prime \prime}={ }^{\top} H \frac{\alpha^{\prime \prime \prime}}{\mathrm{e}\left(\alpha^{\prime \prime \prime}\right)} \mathbb{1}_{\left\{\mathrm{e}\left(\alpha^{\prime \prime \prime}\right) \neq 0\right\}} \cdot X^{\circ} .
$$

Corollary 4.14. The process $\left(X^{\prime}, X^{\prime \prime}, X^{\circ}\right)$ possesses the martingale representation property in $\mathbb{F}$ under $\mathbb{P}$.

Remark 4.15. We note that $\left(X^{\prime}, X^{\prime \prime}, X^{\circ}\right)$ is a locally bounded process. The three processes $X^{\prime}, X^{\prime \prime}, X^{\circ}$ are mutually pathwise orthogonal. The components of the processes $X^{\prime}, X^{\circ}$ are pathwise orthogonal. The path of $X^{\prime \prime}$ is of finite variation. Let $H$ be a $\left(X^{\prime}, X^{\prime \prime}, X^{\circ}\right)$-integrable predictable process. The process $H$ is naturally cut into three parts $\left(H^{\prime}, H^{\prime \prime}, H^{\prime \prime \prime}\right)$ corresponding to $\left(X^{\prime}, X^{\prime \prime}, X^{\circ}\right)$. By the pathwise orthogonality, $H_{h}^{\prime}$ is $X_{h}^{\prime}$-integrable for $1 \leq h \leq d, H^{\prime \prime}$ is $X^{\prime \prime}$-integrable, $H_{h}^{\prime \prime \prime}$ is $X_{h}^{\circ}$-integrable for $1 \leq h \leq \mathrm{n}^{\prime \prime \prime}$.

Theorem 4.2 is proved with $Z^{\prime}=X^{\prime}, Z^{\prime \prime}=X^{\prime \prime}, Z^{\prime \prime \prime}=X^{\circ}$.

\section{FUlly VIABLE MARKET EXPANSION AND THE DRIFT MULTIPLIER ASSUMPTION}

\subsection{The setting}

Let $\mathbb{G}=\left(\mathcal{G}_{t}\right)_{t \geq 0}$ be a second filtration of sub- $\sigma$-algebras of $\mathcal{A}$, containing $\mathbb{F}$, i.e., $\mathcal{G}_{t} \supset \mathcal{F}_{t}$ for $t \geq 0$. We call $\mathbb{G}$ an expansion (or an enlargement) of the filtration $\mathbb{F}$.

We recall the following notion. 
Definition 5.1. Let $T$ be a $\mathbb{G}$ stopping time. We call a strictly positive $\mathbb{G}$ adapted real process $Y$ with $Y_{0}=1$, a local martingale deflator on the time horizon $[0, T]$ for a (multi-dimensional) $(\mathbb{P}, \mathbb{G}$ ) special semimartingale $X$, if the processes $Y$ and $Y X$ are $(\mathbb{P}, \mathbb{G})$ local martingales on $[0, T]$.

This is a notion of no-arbitrage condition. Actually, the existence of local martingale deflators is equivalent to the no-arbitrage conditions NUPBR and $\mathrm{NA}_{1}$ (cf. $[6,16-18,21,23]$ ). We know that, when the no-arbitrage condition NUPBR is satisfied, the market is viable, and vice versa.

We now define rigorously the assumption of the full viability. Let $T$ be a $\mathbb{G}$ stopping time.

Assumption 5.2. (Full viability on $[0, T]$ ) The expansion from $\mathbb{F}$ to $\mathbb{G}$ is fully viable on $[0, T]$. This means that, for any strictly positive $\mathbb{F}$ local martingale $X, X$ has the no-arbitrage property of the first kind in $\mathbb{G}$ on $[0, T]$, i.e. $X$ has a (local martingale) deflator in $\mathbb{G}$ on $[0, T]$.

We are in a situation of an enlargement of filtration. We recall the following fundamental notion (cf. [12,15]).

Assumption 5.3. (Hypothesis $\left(H^{\prime}\right)$ on the time horizon $[0, T]$ ) Every $(\mathbb{P}, \mathbb{F})$ local martingale is a $(\mathbb{P}, \mathbb{G})$ semimartingale on $[0, T]$.

Whenever Hypothesis $\left(H^{\prime}\right)$ holds, the associated drift operator can be defined.

Lemma 5.4. Suppose hypothesis $\left(H^{\prime}\right)$ on $[0, T]$. Then there exists a linear map $\Gamma$ from the space of all $(\mathbb{P}, \mathbb{F})$ local martingales into the space of càdlàg $\mathbb{G}$-predictable processes on $[0, T]$, with finite variation and null at the origin, such that, for any $(\mathbb{P}, \mathbb{F})$ local martingale $X, \tilde{X}:=X-\Gamma(X)$ is a $(\mathbb{P}, \mathbb{G})$ local martingale on $[0, T]$. Moreover, if $X$ is an $\mathbb{F}$ local martingale and $H$ is an $\mathbb{F}$ predictable $X$-integrable process, then $H$ is $\Gamma(X)$-integrable and $\Gamma(H \cdot X)=H \cdot \Gamma(X)$ on $[0, T]$. The operator $\Gamma$ will be called the drift operator.

Proof. Note that, under hypothesis $\left(H^{\prime}\right)$, for any $\mathbb{F}$ local martingale $X, X$ is a special $\mathbb{G}$ semimartingale on $[0, T]$ (cf. [10, Definition 8.4 and Theorem 8.6]) so that the drift operator is well-defined. The linearity of $\Gamma$ is the consequence of the uniqueness of special semimartingale decomposition (cf. [10, Theorem 8.5]). The property of $\Gamma(H \cdot X)$ is the consequence of [14, Lemma 2.2].

In this paper we are interested in the following specific assumption on the drift operator.

Assumption 5.5. (Drift multiplier assumption on $[0, T]$ ) Let $T$ be a $\mathbb{G}$ stopping time.

(1) The Hypothesis $\left(H^{\prime}\right)$ is satisfied on the time horizon $[0, T]$ with a drift operator $\Gamma$.

(2) There exist $N=\left(N_{1}, \ldots, N_{\mathrm{n}}\right)$ an $\mathrm{n}$-dimensional $(\mathbb{P}, \mathbb{F})$ local martingale, and $\varphi$ an $\mathrm{n}$ dimensional $\mathbb{G}$ predictable process such that, for any $(\mathbb{P}, \mathbb{F})$ local martingale $X,[N, X]^{\mathbb{F} \cdot p}$ exists, $\varphi$ is $[N, X]^{\mathbb{F} \cdot p}$-integrable, and

$$
\Gamma(X)={ }^{\top} \varphi \cdot[N, X]^{\mathbb{F}-p}
$$

on the time horizon $[0, T]$.

It is clear that, if $\mathbb{G}$ is fully viable, the hypothesis $\left(H^{\prime}\right)$ from $\mathbb{F}$ to $\mathbb{G}$ is satisfied. This section is devoted to the proof of the following theorem.

Theorem 5.6. Suppose the martingale representation property in $\mathbb{F}$. Suppose the full viability on $[0, T]$. Then, $\Gamma$ satisfies the drift multiplier assumption on $[0, T]$.

\subsection{General consequences of the full viability}

We begin with an immediate consequence of the full viability on the drift operator.

Lemma 5.7. Let $T$ be a $\mathbb{G}$ stopping time. Suppose that the expansion is fully viable on $[0, T]$. For any $\mathbb{F}$ locally bounded local martingale $X$, there exists a strictly positive $\mathbb{G}$ local martingale $Y$ such that

$$
\Gamma(X)=-\frac{1}{Y_{-}} \cdot[Y, X]^{\mathbb{G} \cdot p} \text { on }[0, T]
$$


Proof. For any $\mathbb{F}$ stopping time $T^{\prime}$ such that $X^{T^{\prime}}$ is bounded, for some $a>0, a|\Delta X|<1$ on $\left[0, T^{\prime}\right]$. Let $S=\mathcal{E}(a X)$ which is strictly positive. By the full viability, there exists a strictly positive $\mathbb{G}$ local martingale $Y$ such that $Y S$ is a $\mathbb{G}$ local martingale on $\left[0, T^{\prime} \wedge T\right]$. The lemma is the consequence of the integration by parts formula

$$
\begin{aligned}
& Y S=Y_{0} S_{0}+S_{-} \cdot Y+Y_{-} \cdot S+[Y, S] \text { or equivalently } \\
& a Y_{-} S_{-} \cdot X+a S_{-} \cdot[Y, X]=Y S-Y_{0} S_{0}-S_{-} \cdot Y \text { on }\left[0, T^{\prime} \wedge T\right]
\end{aligned}
$$

Lemma 5.8. Suppose the full viability of the expansion on $[0, T]$. For any $\mathbb{F}$ locally bounded $\mathbb{F}$ optional process $A$ with finite variation, there exists a strictly positive $\mathbb{G}$ local martingale $Y$ such that

$$
(Y \cdot A)^{\mathbb{G} \cdot p}=Y_{-} \cdot A^{\mathbb{F} \cdot p} \quad \text { on }[0, T]
$$

Consequently $A^{\mathbb{G} \cdot p}$ is absolutely continuous with respect to $A^{\mathbb{F} \cdot p}$ on $[0, T]$.

Proof. For any $\mathbb{F}$ stopping time $T^{\prime}>0$ such that $A^{T^{\prime}}$ is bounded, for some $a>0, S=\mathcal{E}\left(a\left(A-A^{\mathbb{F} \cdot p}\right)\right)$ is strictly positive on $\left[0, T^{\prime}\right]$. There exists a strictly positive $\mathbb{G}$ local martingale $Y$ such that $Y S$ is a $\mathbb{G}$ local martingale on $\left[0, T^{\prime} \wedge T\right]$. Write the integration by parts formula

$$
\begin{aligned}
& Y S=Y_{0} S_{0}+S_{-} \cdot Y+Y \cdot S \text { or equivalently } \\
& a Y S_{-} \cdot A-a Y S_{-} \cdot A^{\mathbb{F} \cdot p}=Y S-Y_{0} S_{0}-S_{-} \cdot Y
\end{aligned}
$$

on $\left[0, T^{\prime} \wedge T\right]$. Consequently,

$$
(Y \cdot A)^{\mathbb{G} \cdot p}=\left(Y \cdot A^{\mathbb{F} \cdot p}\right)^{\mathbb{G} \cdot p}={ }^{\mathbb{G} \cdot p}(Y) \cdot A^{\mathbb{F} \cdot p}=Y_{-} \cdot A^{\mathbb{F} \cdot p}
$$

on $\left[0, T^{\prime} \wedge T\right]$. This proves the formula of the lemma.

To prove the absolute continuity of $A^{\mathbb{G} \cdot p}$, without loss of the generality, we assume that $A$ is non decreasing. Let $\mathrm{A}$ be a $\mathbb{G}$ predictable set such that $\mathbb{1}_{\mathrm{A}} \cdot A^{\mathbb{F} \cdot p} \equiv 0$. Then, for any $\epsilon>0$,

$$
\mathbb{E}\left[\mathbb{1}_{\mathrm{A}} \mathbb{1}_{\{Y>\epsilon\}} \cdot A_{T^{\prime} \wedge T}\right] \leq \frac{1}{\epsilon} \mathbb{E}\left[\mathbb{1}_{\mathrm{A}} Y \cdot A_{T^{\prime} \wedge T}\right]=\frac{1}{\epsilon} \mathbb{E}\left[\mathbb{1}_{\mathrm{A}} \cdot(Y \cdot A)_{T^{\prime} \wedge T}^{\mathbb{G} \cdot p}\right]=\frac{1}{\epsilon} \mathbb{E}\left[\mathbb{1}_{\mathrm{A}} Y_{-} \cdot A_{T^{\prime} \wedge T}^{\mathbb{F} \cdot p}\right]=0
$$

so that

$$
\mathbb{E}\left[\mathbb{1}_{\mathrm{A}} \cdot A_{T^{\prime} \wedge T}^{\mathbb{G} \cdot p}\right]=\mathbb{E}\left[\mathbb{1}_{\mathrm{A}} \cdot A_{T^{\prime} \wedge T}\right]=\lim _{\epsilon \downarrow 0} \mathbb{E}\left[\mathbb{1}_{\mathrm{A}} \mathbb{1}_{\{Y>\epsilon\}} \cdot A_{T^{\prime} \wedge T}\right]=0 .
$$

The lemma is proved by [10, Theorem 5.14$]$.

We now apply Lemma 5.8 to view the $\mathbb{F}$ totally inaccessible stopping times.

Corollary 5.9. Suppose the full viability of the expansion on $[0, T]$. For any $\mathbb{F}$ totally inaccessible stopping time $S$, there exists a strictly positive $\mathbb{G}$ local martingale $Y$ such that

$$
\left(Y_{S} \mathbb{1}_{[S, \infty)}\right)^{\mathbb{G} \cdot p}=Y_{-} \cdot\left(\mathbb{1}_{[S, \infty)}\right)^{\mathbb{F} \cdot p}
$$

on $[0, T]$. Consequently $\left(\mathbb{1}_{[S, \infty)}\right)^{\mathbb{G} \cdot p}$ is absolutely continuous with respect to $\left(\mathbb{1}_{[S, \infty)}\right)^{\mathbb{F} \cdot p}$ on $[0, T]$, and $S_{\{S \leq T\}}$ is $\mathbb{G}$ totally inaccessible.

\subsection{Proof of Theorem $\mathbf{5 . 6}$}

Theorem 5.6 can be proved in the following way. Under the martingale representation property, instead of considering every $\mathbb{F}$ local martingale in the formula $(7)$, we only need to consider the components $\left(X_{k}\right)$ of a representation process. Also, to define $N$, we only need to find its coefficients $K_{k}$ in its martingale representation 
$N=\sum_{k} K_{k} \cdot X_{k}$. Consequently, the formula (7) changes into a system of equations in the unknowns $\varphi_{k}$ and $K_{k}$ :

$$
(\nabla \nabla) \quad \Gamma\left(X_{h}\right)=\sum_{k}^{\top} \varphi_{k} K_{k} \cdot\left[X_{k}, X_{h}\right]^{\mathbb{F} \cdot p},
$$

for any components $X_{h}$ of the representation process. (In fact, we could then simply take $N$ to be the representation process.)

To solve the equations $(\nabla \nabla)$, we should link the drifts $\Gamma\left(X_{h}\right)$, for any components $X_{h}$ of the representation process, to the brackets $\left[X_{k}, X_{h}\right]^{\mathbb{E} \cdot p}$. The full viability makes these connections possible, thanks to Lemma 5.7. The computations are further facilitated, because of the representation process $\left(X^{\prime}, X^{\prime \prime}, X^{\circ}\right)$ constructed in Section 4.

\subsubsection{Drift of $X^{\prime}$}

We suppose for the rest of this paper the martingale representation property in $\mathbb{F}$ with a $d$-dimensional representation process and the full viability of the expansion on $[0, T]$. We use the reconstituted representation process $\left(X^{\prime}, X^{\prime \prime}, X^{\circ}\right)$ in Corollary 4.14. We will compute successively the drifts $\Gamma\left(X^{\prime}\right), \Gamma\left(X^{\prime \prime}\right)$ and $\Gamma\left(X^{\circ}\right)$.

We begin with the computation of $\Gamma\left(X^{\prime}\right)$ which is simple.

Lemma 5.10. For $1 \leq h \leq d$, there exists a $\mathbb{G}$ predictable process $G_{h}^{\prime}$ such that

$$
\Gamma\left(X_{h}^{\prime}\right)=G_{h}^{\prime} \cdot\left[X_{h}^{\prime}, X_{h}^{\prime}\right]^{\mathbb{F} \cdot p} \text { on }[0, T] .
$$

Proof. Let $Y$ be defined in Lemma 5.7 for $X_{h}^{\prime}$. By the path continuity, by [3], there exists a $\mathbb{G}$ predictable process $H$ such that

$$
\left[Y, X_{h}^{\prime}\right]^{\mathbb{G} \cdot p}=\left[H \cdot \widetilde{X}_{h}^{\prime}, \widetilde{X}_{h}^{\prime}\right]=H \cdot\left[\widetilde{X}_{h}^{\prime}, \widetilde{X}_{h}^{\prime}\right]=H \cdot\left[X_{h}^{\prime}, X_{h}^{\prime}\right]=H \cdot\left[X_{h}^{\prime}, X_{h}^{\prime}\right]^{\mathbb{F} \cdot p}
$$

on $[0, T]$. Applying Lemma 5.7, we prove the lemma.

\subsubsection{Drift of $X^{\prime \prime}$}

The case of $\Gamma\left(X^{\prime \prime}\right)$ is much more involved. Recall the $\mathbb{F}$ predictable stopping time $T_{n}$ and the partition sets $A_{n, h}$ defined in subsection 4.3 for $1 \leq n<\mathrm{N}^{a}, 0 \leq h \leq d$. Let $\mu$ denote the jump measure of $X^{\prime \prime}$. Let $\nu$ (resp. $\bar{\nu})$ be the $\mathbb{F}$ (resp. $\mathbb{G}$ ) compensator of $\mu$. We consider the stochastic ${ }_{*}$-integral in $\mathbb{F}$ with respect to $(\mu-\nu)$, but also in $\mathbb{G}$ with respect to $(\mu-\bar{\nu})$.

Lemma 5.11. We have $\mathcal{G}(\mathbb{F}, \mu) \mathbb{1}_{[0, T]} \subset \mathcal{G}(\mathbb{G}, \mu)$. For $g \in \mathcal{G}(\mathbb{F}, \mu)$, on $[0, T]$,

$$
g_{*}(\mu-\bar{\nu})=g_{*}(\mu-\nu)-\Gamma\left(g_{*}(\mu-\nu)\right),
$$

and

$$
\Gamma\left(g_{*}(\mu-\nu)\right)=\sum_{n=1}^{\mathrm{N}^{a}-}\left(\mathbb{E}\left[g\left(T_{n}, \Delta_{T_{n}} X^{\prime \prime}\right) \mathbb{1}_{\left\{\Delta_{T_{n}} X^{\prime \prime} \neq 0\right\}} \mid \mathcal{G}_{T_{n}-}\right]-\mathbb{E}\left[g\left(T_{n}, \Delta_{T_{n}} X^{\prime \prime}\right) \mathbb{1}_{\left\{\Delta_{T_{n}} X^{\prime \prime} \neq 0\right\}} \mid \mathcal{F}_{T_{n}-}\right]\right) \mathbb{1}_{\left[T_{n} \infty\right)} .
$$

In particular, $x_{*}(\mu-\bar{\nu})=\widetilde{X}^{\prime \prime}$ on $[0, T]$.

Proof. For $1 \leq n<\mathrm{N}^{a}$, the process

$$
\left(g\left(T_{n}, \Delta_{T_{n}} X^{\prime \prime}\right) \mathbb{1}_{\left\{\Delta_{T_{n}} X^{\prime \prime} \neq 0\right\}}-\mathbb{E}\left[g\left(T_{n}, \Delta_{T_{n}} X^{\prime \prime}\right) \mathbb{1}_{\left\{\Delta_{T_{n}} X^{\prime \prime} \neq 0\right\}} \mid \mathcal{F}_{T_{n}-}\right]\right) \mathbb{1}_{\left[T_{n} \infty\right)}=\mathbb{1}_{\left[T_{n}\right]} \cdot\left(g_{*}(\mu-\nu)\right)
$$


is a $\mathbb{F}$ local martingale. The martingale part in $\mathbb{G}$ of this process is given by

$$
\begin{aligned}
& \left(g\left(T_{n}, \Delta_{T_{n}} X^{\prime \prime}\right) \mathbb{1}_{\left\{\Delta_{T_{n}} X^{\prime \prime} \neq 0\right\}}-\mathbb{E}\left[g\left(T_{n}, \Delta_{T_{n}} X^{\prime \prime}\right) \mathbb{1}_{\left\{\Delta_{T_{n}} X^{\prime \prime} \neq 0\right\}} \mid \mathcal{F}_{T_{n}-}\right]\right) \mathbb{1}_{\left[T_{n} \infty\right)} \\
& -\mathbb{E}\left[\left(g\left(T_{n}, \Delta_{T_{n}} X^{\prime \prime}\right) \mathbb{1}_{\left\{\Delta_{T_{n}} X^{\prime \prime} \neq 0\right\}}-\mathbb{E}\left[g\left(T_{n}, \Delta_{T_{n}} X^{\prime \prime}\right) \mathbb{1}_{\left\{\Delta_{T_{n}} X^{\prime \prime} \neq 0\right\}} \mid \mathcal{F}_{T_{n}-}\right]\right) \mid \mathcal{G}_{T_{n}-}\right] \mathbb{1}_{\left[T_{n} \infty\right)} \\
= & \left(g\left(T_{n}, \Delta_{T_{n}} X^{\prime \prime}\right) \mathbb{1}_{\left\{\Delta_{T_{n}} X^{\prime \prime} \neq 0\right\}}-\mathbb{E}\left[g\left(T_{n}, \Delta_{T_{n}} X^{\prime \prime}\right) \mathbb{1}_{\left\{\Delta_{T_{n}} X^{\prime \prime} \neq 0\right\}} \mid \mathcal{G}_{T_{n}-}\right]\right) \mathbb{1}_{\left[T_{n} \infty\right) .} .
\end{aligned}
$$

(In particular, this shows that $\mathbb{E}\left[g\left(T_{n}, \Delta_{T_{n}} X^{\prime \prime}\right) \mathbb{1}_{\left\{\Delta_{T_{n}} X^{\prime \prime} \neq 0\right\}} \mid \mathcal{G}_{T_{n}-}\right]$ is well-defined.) By Lemma 5.4, this implies also

$$
\begin{aligned}
& \mathbb{1}_{\left[T_{n}\right]} \cdot \Gamma\left(g_{*}(\mu-\nu)\right)=\Gamma\left(\mathbb{1}_{\left[T_{n}\right]} \cdot\left(g_{*}(\mu-\nu)\right)\right) \\
& =\left(\mathbb{E}\left[g\left(T_{n}, \Delta_{T_{n}} X^{\prime \prime}\right) \mathbb{1}_{\left\{\Delta_{T_{n}} X^{\prime \prime} \neq 0\right\}} \mid \mathcal{G}_{T_{n}-}\right]-\mathbb{E}\left[g\left(T_{n}, \Delta_{T_{n}} X^{\prime \prime}\right) \mathbb{1}_{\left\{\Delta_{T_{n}} X^{\prime \prime} \neq 0\right\}} \mid \mathcal{F}_{T_{n}-}\right]\right) \mathbb{1}_{\left[T_{n} \infty\right)}
\end{aligned}
$$

on $[0, T]$. Because $\Gamma\left(g_{*}(\mu-\nu)\right)$ is $\mathbb{G}$ predictable with finite variation on $[0, T]$, the series

$$
\sum_{n=1}^{\mathrm{N}^{a}-}\left|\mathbb{E}\left[g\left(T_{n}, \Delta_{T_{n}} X^{\prime \prime}\right) \mathbb{1}_{\left\{\Delta_{T_{n}} X^{\prime \prime} \neq 0\right\}} \mid \mathcal{G}_{T_{n}-}\right]-\mathbb{E}\left[g\left(T_{n}, \Delta_{T_{n}} X^{\prime \prime}\right) \mathbb{1}_{\left\{\Delta_{T_{n}} X^{\prime \prime} \neq 0\right\}} \mid \mathcal{F}_{T_{n}-}\right]\right| \mathbb{1}_{\left[T_{n} \infty\right)}
$$

is a $\mathbb{G}$ locally integrable predictable process on $[0, T]$. This local integrability, together with the relation

$$
\begin{aligned}
& g\left(T_{n}, \Delta_{T_{n}} X^{\prime \prime}\right) \mathbb{1}_{\left\{\Delta_{T_{n}} X^{\prime \prime} \neq 0\right\}}-\mathbb{E}\left[g\left(T_{n}, \Delta_{T_{n}} X^{\prime \prime}\right) \mathbb{1}_{\left\{\Delta_{T_{n}} X^{\prime \prime} \neq 0\right\}} \mid \mathcal{G}_{T_{n}-}\right] \\
& =g\left(T_{n}, \Delta_{T_{n}} X^{\prime \prime}\right) \mathbb{1}_{\left\{\Delta_{T_{n}} X^{\prime \prime} \neq 0\right\}}-\mathbb{E}\left[g\left(T_{n}, \Delta_{T_{n}} X^{\prime \prime}\right) \mathbb{1}_{\left\{\Delta_{T_{n}} X^{\prime \prime} \neq 0\right\}} \mid \mathcal{F}_{T_{n}-}\right] \\
& +\quad \mathbb{E}\left[g\left(T_{n}, \Delta_{T_{n}} X^{\prime \prime}\right) \mathbb{1}_{\left\{\Delta_{T_{n}} X^{\prime \prime} \neq 0\right\}} \mid \mathcal{F}_{T_{n}-}\right]-\mathbb{E}\left[g\left(T_{n}, \Delta_{T_{n}} X^{\prime \prime}\right) \mathbb{1}_{\left\{\Delta_{T_{n}} X^{\prime \prime} \neq 0\right\}} \mid \mathcal{G}_{T_{n}-}\right],
\end{aligned}
$$

implies $g \mathbb{1}_{[0, T]} \in \mathcal{G}(\mathbb{G}, \mu)$, and also

$$
\begin{aligned}
& \Gamma\left(g_{*}(\mu-\nu)\right)=\Gamma\left(\mathbb{1}_{\cup_{1 \leq n<N^{a}}\left[T_{n}\right]} \cdot\left(g_{*}(\mu-\nu)\right)\right)=\mathbb{1}_{\cup_{1 \leq n<N^{a}\left[T_{n}\right]} \cdot \Gamma\left(g_{*}(\mu-\nu)\right)} \\
= & \sum_{n=1}^{\mathbf{N}^{a}-}\left(\mathbb{E}\left[g\left(T_{n}, \Delta_{T_{n}} X^{\prime \prime}\right) \mathbb{1}_{\left\{\Delta_{T_{n}} X^{\prime \prime} \neq 0\right\}} \mid \mathcal{G}_{T_{n}-}\right]-\mathbb{E}\left[g\left(T_{n}, \Delta_{T_{n}} X^{\prime \prime}\right) \mathbb{1}_{\left\{\Delta_{T_{n}} X^{\prime \prime} \neq 0\right\}} \mid \mathcal{F}_{T_{n}-}\right]\right) \mathbb{1}_{\left[T_{n} \infty\right)}
\end{aligned}
$$

on $[0, T]$. We can now check that $g_{*}(\mu-\bar{\nu})$ and $g_{*}(\mu-\nu)-\Gamma\left(g_{*}(\mu-\nu)\right)$ have the same jumps on $[0, T]$. By [10, Theorem 7.23], they are the same $\mathbb{G}$ local martingales on $[0, T]$.

Remark 5.12. Note that, for $0 \leq h \leq d, X_{h}^{\prime \prime}$ is a bounded process with finite variation. $X_{h}^{\prime \prime}$ is always a $\mathbb{G}$ special semimartingale whatever hypothesis $\left(H^{\prime}\right)$ is valid or not. Denote always by $\widetilde{X}^{\prime \prime}$ the $\mathbb{G}$ martingale part of $X^{\prime \prime}$.

Lemma 5.13. There exist a d-dimensional $\mathbb{F}$ local martingale $N^{\prime \prime}$ of the form $N^{\prime \prime}=H \cdot X^{\prime \prime}$, and a d-dimensional $\mathbb{G}$ predictable process $\varphi^{\prime \prime}$ such that, for every $0 \leq h \leq d,{ }^{\top} \varphi^{\prime \prime} \cdot\left[N^{\prime \prime}, X_{h}^{\prime \prime}\right]^{\mathbb{F} \cdot p}$ exists and

$$
\tilde{X}_{h}^{\prime \prime}=X_{h}^{\prime \prime}-{ }^{\top} \varphi^{\prime \prime} \cdot\left[N^{\prime \prime}, X_{h}^{\prime \prime}\right]^{\mathbb{F} \cdot p}
$$

is a $\mathbb{G}$ local martingale. In particular, in case of the full viability on $[0, T], \Gamma\left(X_{h}^{\prime \prime}\right)={ }^{\top} \varphi^{\prime \prime} \cdot\left[N^{\prime \prime}, X_{h}^{\prime \prime}\right]^{\mathbb{F} \cdot p}$ on $[0, T]$.

Proof. In this proof, we will simply write $N, \varphi$ instead of $N^{\prime \prime}, \varphi^{\prime \prime}$. With the computations in the proof of Lemma 5.11, we know that the $\mathbb{G}$ drift part of $X_{h}^{\prime \prime}$ is given by

$$
\sum_{n=1}^{\mathrm{N}^{a}-} \mathbb{E}\left[\Delta_{T_{n}} X_{h}^{\prime \prime} \mid \mathcal{G}_{T_{n}-}\right] \mathbb{1}_{\left[T_{n} \infty\right)}=\sum_{n=1}^{\mathrm{N}^{a}-} \frac{1}{2^{n}}\left(\mathbb{E}\left[\mathbb{1}_{A_{n, h}} \mid \mathcal{G}_{T_{n}-}\right]-p_{n, h}\right) \mathbb{1}_{\left[T_{n} \infty\right)}=\sum_{n=1}^{\mathrm{N}^{a}-} \frac{1}{2^{n}}\left(\bar{p}_{n, h}-p_{n, h}\right) \mathbb{1}_{\left[T_{n} \infty\right)} .
$$

with $p_{n, h}=\mathbb{E}\left[\mathbb{1}_{A_{n, h}} \mid \mathcal{F}_{T_{n}-}\right]$ and $\bar{p}_{n, h}=\mathbb{E}\left[\mathbb{1}_{A_{n, h}} \mid \mathcal{G}_{T_{n}-}\right]$. We look for a $d$-dimensional $\mathbb{F}$ local martingale $N$ and a $d$-dimensional $\mathbb{G}$ predictable process $\varphi$ such that

$$
\frac{1}{2^{n}}\left(\bar{p}_{n, h}-p_{n, h}\right)={ }^{\top} \varphi_{T_{n}} n_{n, h} p_{n, h},
$$


on $\left\{T_{n}<\infty\right\}$, where $n_{n, h} \in \mathcal{F}_{T_{n}-}$ is the value of $\Delta_{T_{n}} N$ on $A_{n, h}$, or equivalently,

$$
\frac{1}{2^{n}}\left(\frac{\bar{p}_{n, h}}{p_{n, h}}-1\right)={ }^{\top} \varphi_{T_{n}} n_{n, h}, 0 \leq h \leq d,
$$

(with the convention that $\left.\frac{0}{0}-1=0\right)$. Consider the $(1+d)$-dimensional vector $p_{n}=\left(p_{n, h}\right)_{0 \leq h \leq d}$. By GramSchmidt process, we obtain a $\mathcal{F}_{T_{n}-}$ measurable orthonormal basis $\left(\epsilon_{n, 0}, \epsilon_{n, 1}, \epsilon_{n, 2}, \ldots, \epsilon_{n, d}\right)$ in $\mathbb{R} \times \mathbb{R}^{d}$ such that $\epsilon_{n, j}$ is orthogonal to $p_{n}$ for all $1 \leq j \leq d$. Note that

$$
\frac{1}{2^{n}} \sum_{h=0}^{d}\left(\frac{\bar{p}_{n, h}}{p_{n, h}}-1\right) p_{n, h}=\frac{1}{2^{n}} \sum_{h=0}^{d}\left(\bar{p}_{n, h}-p_{n, h}\right)=0 .
$$

This implies that the vector $\frac{1}{2^{n}}\left(\frac{\overline{\mathrm{p}}}{\mathrm{p}}-1\right)$ of the components $\frac{1}{2^{n}}\left(\frac{\bar{p}_{n, h}}{p_{n, h}}-1\right)$ is orthogonal to $p_{n}$ so that it is a linear combination of the $\epsilon_{n, j}, 1 \leq j \leq d$ :

$$
\frac{1}{2^{n}}\left(\frac{\overline{\mathrm{p}}}{\mathrm{p}}-1\right)=\varsigma_{n, 1} \epsilon_{n, 1}+\varsigma_{n, 2} \epsilon_{n, 2}+\ldots+\varsigma_{n, d} \epsilon_{n, d},
$$

where $\varsigma_{n, h}$ are the scalar product of $\frac{1}{2^{n}}\left(\frac{\overline{\mathrm{p}}}{\mathrm{p}}-1\right)$ with $\epsilon_{n, h}$ so that $\mathcal{G}_{T_{n}}$ - measurable. Let ${ }^{\top} \epsilon_{n}$ denote the $d \times(1+d)$ matrix whose lines are the vectors ${ }^{\top} \epsilon_{n, k}, 1 \leq k \leq d$. Let $\varphi_{n}$ denote the vector in $\mathbb{R}^{d}$ of components $2^{n} \varsigma_{n, k}, 1 \leq$ $k \leq d$. Let $n_{n, h}$ denote the $h$ th-column $(0 \leq h \leq d)$ of the matrix ${\frac{1}{2^{n}}}^{\top} \epsilon_{n}$, which is a vector in $\mathbb{R}^{d}$. Then, the above identity becomes

$$
\begin{aligned}
& {\frac{1}{2^{n}}}^{\top}\left(\frac{\overline{\mathrm{p}}}{\mathrm{p}}-1\right)=\sum_{k=1}^{d} \varsigma_{n, k} \epsilon^{\top} \epsilon_{n, k}=\sum_{k=1}^{d} 2^{n} \varsigma_{n, k}{\frac{1}{2^{n}}}^{\top} \epsilon_{n, k}, \\
& \text { or } \\
& \frac{1}{2^{n}}\left(\frac{\bar{p}_{n, h}}{p_{n, h}}-1\right)={ }^{\top} \varphi_{n} n_{n, h}, 0 \leq h \leq d .
\end{aligned}
$$

The equation (8) is solved. We define $d$ number of $\mathbb{F}$ local martingales.

$$
N_{j}={ }^{\top}\left(\sum_{n=1}^{\mathrm{N}^{a}-} \epsilon_{n, j} \mathbb{1}_{\left[T_{n}\right]}\right) \cdot X^{\prime \prime}, 1 \leq j \leq d .
$$

Let $\mathrm{a}_{n}$ denote the vector of the components $\mathbb{1}_{A_{n, h}, 0} \leq h \leq d$. We compute the jumps at $T_{n}<\infty$.

$$
\Delta_{T_{n}} N_{j}={ }^{\top} \epsilon_{n, j} \Delta_{T_{n}} X^{\prime \prime}=\frac{1}{2^{n}}{ }^{\top} \epsilon_{n, j}\left(\mathrm{a}_{n}-p_{n}\right)={\frac{1}{2^{n}}}^{\top} \epsilon_{n, j} \mathrm{a}_{n}, 1 \leq j \leq d
$$

Hence, if $\varphi=\sum_{n=1}^{\mathrm{N}^{a}-} 2^{n} \varphi_{n} \mathbb{1}_{\left[T_{n}\right]}$, for $0 \leq h \leq d$,

$$
\begin{aligned}
& { }^{\top} \varphi \cdot\left[N, X_{h}^{\prime \prime}\right]^{\mathbb{F} \cdot p}=\sum_{n=1}^{\mathrm{N}^{a}-} 2^{n \top} \varphi_{n} \mathbb{E}\left[\Delta_{T_{n}} N \Delta_{T_{n}} X_{h}^{\prime \prime} \mid \mathcal{F}_{T_{n}-}\right] \mathbb{1}_{\left[T_{n}, \infty\right)} \\
= & \sum_{n=1}^{\mathrm{N}^{a}-} 2^{n \top} \varphi_{n} \mathbb{E}\left[\frac{1}{2^{n}} \epsilon_{n} \mathrm{a}_{n} \Delta_{T_{n}} X_{h}^{\prime \prime} \mid \mathcal{F}_{T_{n}-}\right] \mathbb{1}_{\left[T_{n}, \infty\right)} \\
= & \sum_{n=1}^{\mathrm{N}^{a}-} 2^{n} \sum_{j=0}^{\top}{ }^{\top} \varphi_{n} \mathbb{E}\left[\frac{1}{2^{n}} \epsilon_{n} \boldsymbol{a}_{n} \Delta_{T_{n}} X_{h}^{\prime \prime} \mathbb{1}_{A_{n, j}} \mid \mathcal{F}_{T_{n}-}\right] \mathbb{1}_{\left[T_{n}, \infty\right)} \\
= & \sum_{n=1}^{\mathrm{N}^{a}-} 2^{n} \sum_{j=0}^{d} \varphi_{n} \varphi_{n, j} x_{n, j} \mathbb{E}\left[\mathbb{1}_{A_{n, j}} \mid \mathcal{F}_{T_{n}-}\right] \mathbb{1}_{\left[T_{n}, \infty\right)} \\
& \text { where } x_{n, j}=\frac{1}{2^{n}}\left(\delta_{h, j}-p_{n, h}\right) \text { is the value of } \Delta_{T_{n}} X_{h}^{\prime \prime} \text { on } A_{n, j}, \\
= & \sum_{n=1}^{\mathrm{N}^{a}-} 2^{n} \sum_{j=0}^{d} \varphi_{n} n_{n, j} x_{n, j} p_{n, j} \mathbb{1}_{\left[T_{n}, \infty\right)} \\
= & \sum_{n=1}^{\mathrm{N}^{a}-1} 2^{n} \sum_{j=0}^{d} \frac{1}{2^{n}}\left(\frac{\bar{p}_{n, j}}{p_{n, j}}-1\right) x_{n, j} p_{n, j} \mathbb{1}_{\left[T_{n}, \infty\right)} \\
= & \sum_{n=1}^{\mathrm{N}^{a}-} \sum_{j=0}^{d} \frac{1}{2^{n}}\left(\bar{p}_{n, j}-p_{n, j}\right)\left(\delta_{h, j}-p_{n, h}\right) \mathbb{1}_{\left[T_{n}, \infty\right)} \\
= & \sum_{n=1}^{\mathrm{N}^{a}-1} \frac{1}{2^{n}}\left(\bar{p}_{n, h}-p_{n, h}\right) \mathbb{1}_{\left[T_{n}, \infty\right)} \\
= & \mathbb{G} \text { drift part of } X_{h}^{\prime \prime} .
\end{aligned}
$$


(Modifying a little the above computation, we can prove that the stochastic integral ${ }^{\top} \varphi \cdot\left[N, X_{h}^{\prime \prime}\right]^{\mathbb{F} \cdot p}$ exists.)

The following lemma will not be used in this paper, but essential to solve the accessible raw structure equation (4) in $[20]$.

Lemma 5.14. For $1 \leq n<\mathrm{N}^{a}$, let $\mathrm{I}_{n}=\left\{0 \leq h \leq d: p_{n, h}>0\right\}$. The kernel of the matrix $\Delta_{T_{n}}\left[X^{\prime \prime},{ }^{\top} X^{\prime \prime}\right]^{\mathbb{F} \cdot p}$ on $\left\{T_{n}<\infty\right\}$ is

$$
\left\{a \in \mathbb{R} \times \mathbb{R}^{d}: a_{h} \text { is constant on } h \in \mathrm{I}_{n}\right\} .
$$

There exists a $\mathbb{G}$ predictable matrix valued process $G$ such that $\left[\tilde{X}^{\prime \prime},{ }^{\top} \tilde{X}^{\prime \prime}\right]^{\mathbb{G} \cdot p}=G \cdot\left[X^{\prime \prime},{ }^{\top} X^{\prime \prime}\right]^{\mathbb{F} \cdot p}$.

Proof. Fix $1 \leq n<\mathrm{N}^{a}$. $\mathrm{I}_{n}$ is an $\mathcal{F}_{T_{n}-}$ measurable random variable. For an example, suppose $\mathrm{I}_{n}=$ $\{0, \ldots, k\}$. Let a denote the vector of the $\mathbb{1}_{A_{n, h}, 0} \leq h \leq k$, and $p$ denote the vector of the $p_{n, h}, 0 \leq h \leq k$. We write

$$
\begin{aligned}
& \left(\mathbb{E}\left[\Delta_{T_{n}} X_{i}^{\prime \prime} \Delta_{T_{n}} X_{j}^{\prime \prime} \mid \mathcal{F}_{T_{n}-}\right]\right)_{1 \leq i, j \leq k}=\frac{1}{4^{n}} \mathbb{E}\left[(\mathrm{a}-p)^{\top}(\mathrm{a}-p) \mid \mathcal{F}_{T_{n}-}\right] \mathbb{1}_{\left[T_{n}, \infty\right)} \\
= & \frac{1}{4^{n}} \mathbb{E}\left[\mathrm{a}^{\top} \mathrm{a}-\mathrm{a}^{\top} p-p^{\top} \mathrm{a}+p^{\top} p \mid \mathcal{F}_{T_{n}-}\right] \mathbb{1}_{\left[T_{n}, \infty\right)} \\
= & \frac{1}{4^{n}}\left(\mathfrak{D}_{p}-p^{\top} p\right) \mathbb{1}_{\left[T_{n}, \infty\right)}
\end{aligned}
$$

where $\mathfrak{D}_{p}$ denotes the diagonal matrix of diagonal vector $p$. For any vector $a=\left(a_{0}, \ldots, a_{k}\right)$, if

$$
0={ }^{\top} a\left(\mathfrak{D}_{p}-p^{\top} p\right) a=\mathbb{E}\left[\left({ }^{\top} a \mathrm{a}-{ }^{\top} a p\right)^{2} \mid \mathcal{F}_{T_{n}-}\right]
$$

necessarily $\left({ }^{\top} a \mathrm{a}-{ }^{\top} a p\right)^{2}=0$ or $a_{h}={ }^{\top} a p$ for all $1 \leq h \leq k$. This means that the kernel of $\left(\mathfrak{D}_{p}-p^{\top} p\right)$ is the vector space $\mathcal{K}$ generated by the vector $(1,1, \ldots, 1) \in \mathbb{R}^{k}$, while its image space, as $\left(\mathfrak{D}_{p}-p^{\top} p\right)$ is symmetric, is $\mathcal{K}^{\perp} \subset \mathbb{R}^{k}$. The matrix $\left(\mathfrak{D}_{p}-p^{\top} p\right)$ as an operator on $\mathcal{K}^{\perp}$ is invertible. This implies the existence of an $\mathcal{F}_{T_{n}-}$ measurable matrix $\mathrm{J}$ such that $\mathrm{J}\left(\mathfrak{D}_{p}-p^{\top} p\right)$, on $\left\{\mathrm{I}_{n}=\{1, \ldots, k\}\right\} \cap\left\{T_{n}<\infty\right\}$, is the projection operator onto the space $\mathcal{K}^{\perp}$.

We can make the same analysis with $\left[\tilde{X}^{\prime \prime},{ }^{\top} \tilde{X}^{\prime \prime}\right]^{\mathbb{G} \cdot p}$. Notice that $\Delta_{T_{n}} \tilde{X}_{h}^{\prime \prime}=\frac{1}{2^{n}}\left(\mathbb{1}_{A_{n, h}}-\bar{p}_{n, h}\right) \mathbb{1}_{\left[T_{n}, \infty\right)}$. Notice that, on the set $\left\{\mathrm{I}_{n}=\{1, \ldots, k\}\right\} \cap\left\{T_{n}<\infty\right\}, \bar{p}_{n, h}=0$ for $h>k$. We obtain then that the vector $(1,1, \ldots, 1) \in$ $\mathbb{R}^{k}$ is in the kernel of the matrix

$$
\mathbf{M}:=\left(\mathbb{E}\left[\Delta_{T_{n}} \tilde{X}_{i}^{\prime \prime} \Delta_{T_{n}} \tilde{X}_{j}^{\prime \prime} \mid \mathcal{G}_{T_{n}-}\right]\right)_{1 \leq i, j \leq k}
$$

and by the symmetry, the image of the $\mathbf{M}$ is contained in $\mathcal{K}^{\perp}$. Now, for any vector $a \in \mathcal{K}, \mathbf{M} a=0=$ $\operatorname{MJ}\left(\mathfrak{D}_{p}-p^{\top} p\right) a$, while for any vector $a \in \mathcal{K}^{\perp}, \mathbf{M} a=\mathbf{M J}\left(\mathfrak{D}_{p}-p^{\top} p\right) a$, proving $\mathbf{M}=\mathbf{M J}\left(\mathfrak{D}_{p}-p^{\top} p\right)$. Finally,

$$
\begin{aligned}
\Delta_{T_{n}}\left[\widetilde{X}^{\prime \prime},{ }^{\top} \tilde{X}^{\prime \prime}\right]^{\mathbb{G} \cdot p} & =\left(\begin{array}{cc}
\mathbf{M}, & 0 \\
0, & 0
\end{array}\right)=\left(\begin{array}{rr}
\mathbf{M J}\left(\mathfrak{D}_{p}-p^{\top} p\right), & 0 \\
0 & \\
\mathbf{M}, & 0 \\
0, & 0
\end{array}\right)\left(\begin{array}{rr}
\mathrm{J} & 0 \\
0, & 0
\end{array}\right)\left(\begin{array}{cc}
\mathfrak{D}_{p}-p^{\top} p, & 0 \\
0, & 0
\end{array}\right) \\
& =\Delta_{T_{n}}\left[\tilde{X}^{\prime \prime},{ }^{\top} \tilde{X}^{\prime \prime}\right]^{\mathbb{G} \cdot p}\left(\begin{array}{ll}
\mathrm{J} & 0 \\
0, & 0
\end{array}\right) 4^{n} \Delta_{T_{n}}\left[X^{\prime \prime},{ }^{\top} X^{\prime \prime}\right]^{\mathbb{F} \cdot p}
\end{aligned}
$$

on $\left\{\mathrm{I}_{n}=\{1, \ldots, k\}\right\} \cap\left\{T_{n}<\infty\right\}$. On this set, define

$$
J_{n}=\left(\begin{array}{cc}
\mathrm{J}, & 0 \\
0, & 0
\end{array}\right) 4^{n} .
$$


Now, making the above computation on the set $\left\{\mathrm{I}_{n}=\mathrm{B}\right\} \cap\left\{T_{n}<\infty\right\}$ for any no-empty subset $\mathrm{B}$ of $\{1, \ldots, d\}$ (instead of $\{1, \ldots, k\}$ ), we obtain an $\mathcal{F}_{T_{n}}$ - measurable matrix valued random variable everywhere defined $J_{n}$ such that

$$
\Delta_{T_{n}}\left[\tilde{X}^{\prime \prime},{ }^{\top} \tilde{X}^{\prime \prime}\right]^{\mathbb{G} \cdot p}=\Delta_{T_{n}}\left[\tilde{X}^{\prime \prime},{ }^{\top} \tilde{X}^{\prime \prime}\right]^{\mathbb{G} \cdot p} J_{n} \Delta_{T_{n}}\left[X^{\prime \prime},{ }^{\top} X^{\prime \prime}\right]^{\mathbb{F} \cdot p} .
$$

The lemma is proved with

$$
G=\sum_{n=1}^{\mathrm{N}^{a}-} \Delta_{T_{n}}\left[\tilde{X}^{\prime \prime},{ }^{\top} \tilde{X}^{\prime \prime}\right]^{\mathbb{G} \cdot p} J_{n} \mathbb{1}_{\left[T_{n}\right]}
$$

\subsubsection{Drift of $X_{h}^{\circ}$}

For $1 \leq h \leq \mathrm{n}^{\prime \prime \prime}$, Let $\mu$ denote the jump measure of $X_{h}^{\circ}$. Let $\nu$ (resp. $\bar{\nu}$ ) be the $\mathbb{F}$ (resp. $\mathbb{G}$ ) compensator of $\mu$.

Lemma 5.15. We have $\mathcal{G}(\mathbb{F}, \mu) \mathbb{1}_{[0, T]} \subset \mathcal{G}(\mathbb{G}, \mu)$. Let $g \in \mathcal{G}(\mathbb{F}, \mu)$. Then, on $[0, T], \Gamma\left(g_{*}(\mu-\nu)\right)$ is continuous and

$$
g_{*}(\mu-\bar{\nu})=g_{*}(\mu-\nu)-\Gamma\left(g_{*}(\mu-\nu)\right) .
$$

In particular, $x_{*}(\mu-\bar{\nu})=\widetilde{X}_{h}^{\circ}$ on $[0, T]$.

Proof. According to Lemma 5.9, the support set of $\mu$ avoids any $\mathbb{G}$ predictable stopping time $U$ on $[0, T]$ so that $\mathbb{1}_{[U] *} \bar{\nu}=0$ on $[0, T]$. This implies $\mathcal{G}(\mathbb{F}, \mu) \mathbb{1}_{[0, T]} \subset \mathcal{G}(\mathbb{G}, \mu)$ (cf. [10, Definition 11.16]). On the other hand, $X=g_{*}(\mu-\nu)-\Gamma\left(g_{*}(\mu-\nu)\right)$ is a $\mathbb{G}$ local martingale on $[0, T]$, whose jump $\Delta_{U} X$ at the $\mathbb{G}$ predictable stopping time $U$ is given by $-\Delta_{U} \Gamma\left(g_{*}(\mu-\nu)\right)$ on $\{U \leq T\}$ which is $\mathcal{G}_{U-}$ measurable. Hence, $\Delta_{U} X=\Delta_{U} \Gamma\left(g_{*}(\mu-\nu)\right)=0$ on $\{U \leq T\}$ (cf. [10, Theorem 7.13]), i.e. $\Gamma\left(g_{*}(\mu-\nu)\right)$ is continuous on $[0, T]$. Now we compute the jumps on $[0, T]$.

$$
\Delta_{s}\left(g_{*}(\mu-\bar{\nu})\right)=g\left(s, \Delta_{s} X_{h}^{\circ}\right) \mathbb{1}_{\left\{\Delta_{s} X_{h}^{\circ} \neq 0\right\}}=\Delta_{s}\left(g_{*}(\mu-\nu)-\Gamma\left(g_{*}(\mu-\nu)\right)\right) .
$$

The lemma is proved by [10, Theorem 7.23].

Lemma 5.16. There exists a $\mathbb{G}$ predictable process $G_{h}^{\circ}$ such that, on $[0, T]$,

$$
\Gamma\left(X_{h}^{\circ}\right)=G_{h}^{\circ} \cdot\left[X_{h}^{\circ}, X_{h}^{\circ}\right]^{\mathbb{F} \cdot p} .
$$

on $[0, T]$.

Proof. Let $Y$ be a $\mathbb{G}$ local martingale defined in Lemma 5.7 for $X_{h}^{\circ}$. By Lemma 3.9, there exists $g \in \mathcal{G}(\mathbb{G}, \mu)$ such that, on $[0, T]$,

$$
\left[Y, X_{h}^{\circ}\right]^{\mathbb{G} \cdot p}=\left[Y, \widetilde{X}_{h}^{\circ}\right]^{\mathbb{G} \cdot p}=\left[g_{*}(\mu-\bar{\nu}), \widetilde{X}_{h}^{\circ}\right]^{\mathbb{G} \cdot p} .
$$

Using the notations of Section 4.5, we verify that the time support of $\mu$ is

$$
\left\{e_{h}\left(\alpha_{h}^{\prime \prime \prime}\right) \mathbb{1}_{\left\{\Delta X^{\prime \prime \prime}=\alpha_{h}^{\prime \prime \prime} \neq \mathbf{0}\right\}} \neq 0\right\}=\left\{\Delta X^{\prime \prime \prime}=\alpha_{h}^{\prime \prime \prime} \neq \mathbf{0}\right\},
$$

while its space location process can be $e_{h}\left(\alpha_{h}^{\prime \prime \prime}\right)$. We see that $\mu$ satisfies the finite $\mathbb{F}$ predictable constraint condition (with constraint process $e_{h}\left(\alpha_{h}^{\prime \prime \prime}\right)$ ) and satisfies the conditions in Theorem 3.8 on $[0, T]($ with $\mathrm{n}=1)$. Hence, there exists a $\mathbb{G}$ predictable process $H$ such that

$$
\left[g_{*}(\mu-\bar{\nu}), \widetilde{X}_{h}^{\circ}\right]^{\mathbb{G} \cdot p}=\left[H \cdot \widetilde{X}_{h}^{\circ}, \widetilde{X}_{h}^{\circ}\right]^{\mathbb{G} \cdot p}=H \cdot\left[X_{h}^{\circ}, X_{h}^{\circ}\right]^{\mathbb{G} \cdot p}
$$

on $[0, T]$. By Lemma $5.8,\left[X_{h}^{\circ}, X_{h}^{\circ}\right]^{\mathbb{G} \cdot p}$ is absolutely continuous with respect to $\left[X_{h}^{\circ}, X_{h}^{\circ}\right]^{\mathbb{F} \cdot p}$ on $[0, T]$. Let

$$
K=\frac{\mathrm{d}\left[X_{h}^{\circ}, X_{h}^{\circ}\right]^{\mathbb{G} \cdot p}}{\mathrm{~d}\left[X_{h}^{\circ}, X_{h}^{\circ}\right]^{\mathbb{F} \cdot p}}
$$


Then, on $[0, T]$,

$$
\left[Y, X_{h}^{\circ}\right]^{\mathbb{G} \cdot p}=H \cdot\left[X_{h}^{\circ}, X_{h}^{\circ}\right]^{\mathbb{G} \cdot p}=H K \cdot\left[X_{h}^{\circ}, X_{h}^{\circ}\right]^{\mathbb{F} \cdot p} .
$$

Together with Lemma 5.7, this concludes the proof.

\subsubsection{The final proof}

Proof of Theorem 5.6. In fact, for any $\mathbb{F}$ local martingale $X$, we write $X$ in its martingale representation $H^{\prime} \cdot X^{\prime}+H^{\prime \prime} \cdot X^{\prime \prime}+H^{\circ} \cdot X^{\circ}$ with respect to the representation process $\left(X^{\prime}, X^{\prime \prime}, X^{\circ}\right)$ (cf. Remark 4.15). Let A be any $\mathbb{F}$ predictable set such that $H^{\prime} \mathbb{1}_{\mathrm{A}}, H^{\prime \prime} \mathbb{1}_{\mathrm{A}}, H^{\circ} \mathbb{1}_{\mathrm{A}}$ are bounded. By Lemma 5.10, Lemma 5.13, Lemma 5.16, Lemma 5.4 and Remark 4.15, we compute, on $[0, T]$,

$$
\begin{aligned}
& \mathbb{1}_{\mathrm{A}} \cdot \Gamma(X)=\Gamma\left(\mathbb{1}_{\mathrm{A}} \cdot X\right) \\
= & \Gamma\left(\mathbb{1}_{\mathrm{A}} H^{\prime} \cdot X^{\prime}\right)+\Gamma\left(\mathbb{1}_{\mathrm{A}} H^{\prime \prime} \cdot X^{\prime \prime}\right)+\Gamma\left(\mathbb{1}_{\mathrm{A}} H^{\circ} \cdot X^{\circ}\right)(\text { Remark 4.15) } \\
= & \sum_{h} \Gamma\left(\mathbb{1}_{\mathrm{A}} H_{h}^{\prime} \cdot X_{h}^{\prime}\right)+\Gamma\left(\mathbb{1}_{\mathrm{A}} H^{\prime \prime} \cdot X^{\prime \prime}\right)+\sum_{h} \Gamma\left(\mathbb{1}_{\mathrm{A}} H_{h}^{\circ} \cdot X_{h}^{\circ}\right) \text { (Remark 4.15) } \\
= & \sum_{h} \mathbb{1}_{\mathrm{A}} H_{h}^{\prime} \cdot \Gamma\left(X_{h}^{\prime}\right)+\mathbb{1}_{\mathrm{A}} H^{\prime \prime} \cdot \Gamma\left(X^{\prime \prime}\right)+\sum_{h} \mathbb{1}_{\mathrm{A}} H_{h}^{\circ} \cdot \Gamma\left(X_{h}^{\circ}\right)(\text { Lemma 5.4) } \\
= & \sum_{h} \mathbb{1}_{\mathrm{A}} H_{h}^{\prime} G_{h}^{\prime} \cdot\left[X_{h}^{\prime}, X_{h}^{\prime}\right]^{\mathbb{F} \cdot p}+\mathbb{1}_{\mathrm{A}} H^{\prime \prime} \varphi^{\prime \prime} \cdot\left[N^{\prime \prime}, X_{h}^{\prime \prime}\right]^{\mathbb{F} \cdot p}+\sum_{h} \mathbb{1}_{\mathrm{A}} H_{h}^{\circ} G_{h}^{\circ} \cdot\left[X_{h}^{\circ}, X_{h}^{\circ}\right]^{\mathbb{F} \cdot p} \\
& (\text { Lemma } 5.10, \text { Lemma 5.13, Lemma } 5.16) \\
= & \sum_{h} \mathbb{1}_{\mathrm{A}} G_{h}^{\prime} \cdot\left[X_{h}^{\prime}, H_{h}^{\prime} \cdot X_{h}^{\prime}\right]^{\mathbb{F} \cdot p}+\mathbb{1}_{\mathrm{A}}^{\top} \varphi^{\prime \prime} \cdot\left[N^{\prime \prime}, H_{h}^{\prime \prime} \cdot X_{h}^{\prime \prime}\right]^{\mathbb{F} \cdot p}+\sum_{h} \mathbb{1}_{\mathrm{A}} G_{h}^{\circ} \cdot\left[X_{h}^{\circ}, H_{h}^{\circ} \cdot X_{h}^{\circ}\right]^{\mathbb{F} \cdot p} \\
= & \sum_{h} \mathbb{1}_{\mathrm{A}} G_{h}^{\prime} \cdot\left[X_{h}^{\prime}, X\right]^{\mathbb{F} \cdot p}+\mathbb{1}_{\mathrm{A}}^{\top} \varphi^{\prime \prime} \cdot\left[N^{\prime \prime}, X\right]^{\mathbb{F} \cdot p}+\sum_{h} \mathbb{1}_{\mathrm{A}} G_{h}^{\circ} \cdot\left[X_{h}^{\circ}, X\right]^{\mathbb{F} \cdot p} \cdot
\end{aligned}
$$

This proves firstly the integrability conditions and then Theorem 5.6.

\section{ACKNOWLEDGMENTS}

This research has benefited from the support of the Chaire Markets in Transition from French Banking Federation.

\section{REFERENCES}

[1] Acciaio B. and Fontana C. and Kardaras C. (2014) "Arbitrage of the first kind and filtration enlargements in semimartingale financial models" arXiv:1401.7198

[2] Aksamit A. and Choulli T. and Deng J. and Jeanblanc M. (2013) "Non-arbitrage up to random horizons for semimartingale models" arXiv:1310.1142

[3] Ansel J. and Stricker C. (1993) "Décomposition de Kunita-Watanabe" Séminaire de Probabilités $2730-32$

[4] Bharucha-Reid A. and Sambandham M. Random Polynomials Academic Press 1986

[5] Barlow M. and Emery M. and Knight F.B. and Song S. and Yor M. (1998) "Autour d'un thorme de Tsirelson sur des filtrations browniennes et non browniennes" Sminaire de Probabilits 32 264-305

[6] Choulli T. and Deng J. and Ma J. (2015) "How nonarbitrage, viability and numraire portfolio are related" Finance and Stochastics 19 719-741

[7] Davis M. "Martingale representation and all that" in: System and Control: Foundation and Applications Springer 2005

[8] Fontana, C. and Jeanblanc M. And Song S. (2014) "On arbitrages arising with honest times" Finance and Stochastics 18 $515-543$

[9] Grorud A. And Pontier M. (1998) Insider trading in a continuous time market model. International Journal of Theoretical and Applied Finance 1 331-347

[10] He, S.W. And Wang, J.G. And Yan, J.A. Semimartingale Theory and Stochastic Calculus, Science Press and CRC Press, Beijing 1992

[11] JACOD J. Calcul stochastique et problmes de martingales Springer-Verlag 1979

[12] JACOD J. (1985) "Grossissement initial, hypothse $\left(H^{\prime}\right)$ et thorme de Girsanov" in: Jeulin T. and Yor M. (eds.) Grossissements de filtrations: exemples et applications Lecture Notes in Mathematics 1118, Springer-Verlag 15-35

[13] Jacod, J. And Shiryaev, A.N. Limit Theorems for Stochastic Processes, second edition, Springer, Berlin - Heidelberg - New York 2003

[14] Jeanblanc, M. And Song, S. (2015) "Martingale representation property in progressively enlarged filtrations" Stochastic Processes and their Applications 125(11) 4242-4271 
[15] Jeulin T. Semi-martingales et grossissement d'une filtration. Lecture Notes in Mathematics, 833, Springer 1980

[16] Kabanov Y.M. (1997) "On the FTAP of Kreps-Delbaen-Schachermayer" in: Kabanov Y.M. and Rozovskii B.L. and Shiryaev A.N. (eds.) Statistics and control of stochastic processes Moscow World Sci. Publ. River Edge 1995/1996 191-203

[17] Karatzas, I. And Kardaras, K. (2007) "The numeraire portfolio in semimartingale financial models" Finance and Stochastics $11447-493$

[18] Kardaras C. (2012) "Market viability via absence of arbitrage of the first kind" Finance and Stochastics 16 651-667

[19] Song S. (2012) "Drift operator in a market affected by the expansion of information flow: a case study" arXiv:1207.1662v1

[20] Song S. (2016) "Drift operator in a viable expansion of information flow" Stochastic Processes and Applications 126(8) $2297-2322$

[21] Song S. (2013) "An alternative proof of a result of Takaoka" arXiv 1306.1062

[22] Song S. (2015) "Construction of multi-default models with full viability" arXiv:1511.00331v1

[23] Schweizer M. and TAKaOKa K. (2014) "A note on the condition of no unbounded profit with bounded risk" Finance and Stochastics 18 393-405 